\title{
Dynamical Analysis of Corporate Bonds based on the Yield Spread Term-Quality Surface
}

\author{
Tomoaki Shouda* \\ Mitsubishi UFJ Trust Investment Technology Institute Co., Ltd. 2-5-6 Shiba, \\ Minato-ku, Tokyo, 105-0014 Japan Tel : +81-3-3457-1977 Fax : +81-3-3457-0910 \\ and Graduate School of International Corporate Strategy, Hitotsubashi University \\ 2-1-2 Hitotsubashi, Chiyoda-ku, Tokyo 101-8439 Japan \\ e-mail: tshouda@ics.hit-u.ac.jp
}

January 25, 2005, this version January 16, 2006

\begin{abstract}
Our aim of this research is to propose a model which estimates implied relative credit reliability from the yield spread of defaultable bonds and evaluates their spread risk. We introduce "Yield Spread Term-Quality Surface" (YSTQS) which is defined on the space of duration and credit reliability of the issuers, and express their yield spread. First, we review the general pricing theorem of defaultable bonds with unpredictable recovery in the no-arbitrage context based on the external hazard rates. Second, we show that the dynamics of state variables determine the shape of the YSTQS, and they drive the YSTQS if the loss-adjusted hazard rates are described by a function of them. Finally, we show an empirical analysis of our model with daily yield spread, duration, and the credit ratings of corporate bonds.
\end{abstract}

Keywords: Default risk, Hazard rate, Yield spread surface, Credit quality, Spread risk, Markov state variable, No-arbitrage

JEL Classifications: C32, C33, C51, G33

\section{Introduction}

Credit risk is the potential loss of obligees' fortune caused by the default of their obligors. Among the credit risk, the fluctuation of the claim's price in the secondary market caused by the deterioration of the obligors' credit reliabilities is called spread risk. The evaluation of fair spread and spread risk are as much important as the default probability itself to the investors of defaultable assets.

Our aim of this research is to propose a model which estimates implied relative credit reliability from the yield spread of defaultable bonds and evaluates their spread risk. And to associate their spread risk, it can describe defaults of many entities at same time.

\footnotetext{
* The author thanks NAKAMURA Nobuhiro, associate professor of Hitotsubashi University, and NAKAGAWA Hidetoshi, associate professor of Tokyo Institute of Technology, for their accurate advises. And we also thank Monique Jeanblanc, professor of Evry University, for stimulating discussions and insightful comments. The theoretical part of this article is originally introduced in Shouda (2005).
} 
There exist several approaches to analyze credit risk. Some kind of static analysis, like discriminate analysis with accounting information, is often used in the financial decision making. But to evaluate spread risk, dynamical analyses are suitable. Structural approach is the one of them. It is the direct approach to model the default mechanism. In the structural approaches, companies will default when their liability exceed their repayment ability (Merton (1974), Black and Cox (1976)).

On the other hand, default times are modeled as external stopping times with their intensities (hazard rates) in the reduced approach. Thanks to the flexibility and affinity of hazard rates with the credit yield spreads, this approach is advantageous to evaluate credit derivatives.

The models based on reduced approach are roughly divided into the following two kinds. The first is formulated by a Markov chain with stochastic variables defined on some discrete space (Jarrow, Lando and Turnbull (1997)). They are often regarded as the credit ratings. The key concept of them is the rating transition matrix which express the migration probability of credit ratings. The drawback of this kind of models to describe plural entities is explosion of rating transition matrix caused by the naive expansions to evaluate joint default probability of them. To avoid this difficulty, conditionally independent migration is often assumed. Farnsworth and Li (2003) demonstrated an empirical analysis of plural entities with an extended rating transition matrix.

The second is formulated by stochastic processes as the default intensities. Duffie and Singleton (1999) showed that if the expected loss is expressed as a fraction of pre-default price, the defaultable discount bonds are priced by the discount rate which is composed of the default free interest rate and the loss-adjusted hazard rate. The lossadjusted hazard rate is the instantaneous unconditional expected loss rate. Kusuoka (1999) pointed out that we must care about the filtration to apply reduced model to the defaults of plural entities. Duffie and Lando (2001) showed an important research about the relation between reduced and structural model that if obligees know obligors' accounting information partially, reduced models are derived from structural models. Duffee (1999) demonstrated an individual company-wise empirical analysis of an intensity based model with the extended Kalman filter.

The essential problem with the evaluation of bonds is to estimate their yield. And they are associated to the yield term structure for each issuer.

See figure 1 which shows yield spread of corporate bonds by their duration at September 30, 2005. The markers distinguish each credit rating. We can see some relation between the yield spreads and the 
credit ratings, but it is difficult to draw one 'curve' which contains all of yield spreads and duration points for each credit rating.

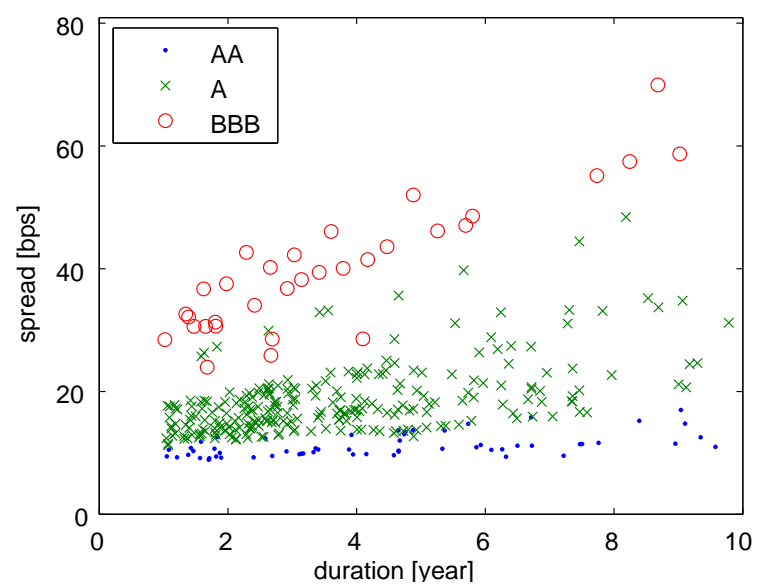

Figure 1. Yield spread of corporate bonds by their duration at September 30, 2005.

Figure 2 shows the distribution of durations, credit ratings and yield spreads historically. It also shows the sector classification of the issuers. We find that the disutribusion of duration and credit ratings are stable, but the one of yield spread is not. It is required to describe these facts to our model.

The following two approaches are well known to formulate the dynamics of yield term structure of default free bonds which hold the no arbitrage principle.

The first is the Markov state variable models. The affine types (Duffie and Kan (1996)) and the Quadratic Gaussian types (Constantindes (1992), Jamshidian (1996)) are representative of them. The characteristics of this kind of models are that the yield term structure is represented by the function of finite Markov state variables, and the partial differential equations which the function must hold are derived. But in those models, the shape of yield term structure is restricted and it is difficult to capture the actual market.

The second is the forward rate models introduced by Heath, Jarrow and Morton (1992). They modeled the dynamics of forward rates directly, and show that the drifts in the risk neutral measure are determined by the no arbitrage principle. In this kind of models, the current term structure is taken as the initial value of the model. But the implementation is rather difficult other than the Gaussian type models.

In this research, we construct an intensity based model with Markov state variables. The loss-adjusted hazard rates on the risk neutral mea- 


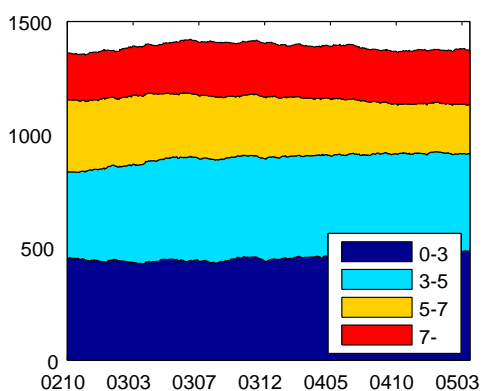

Number of bonds in each duration sector (historical path).

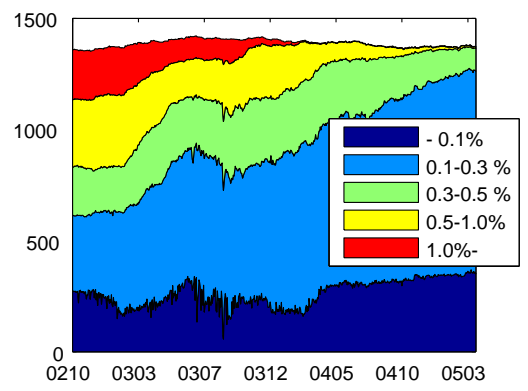

Number of bonds in each spread sector (historical path).

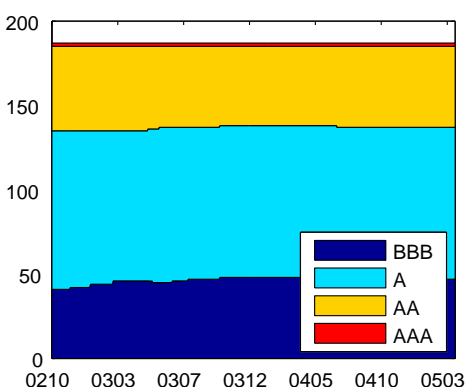

Number of entities in each credit rating (historical path).

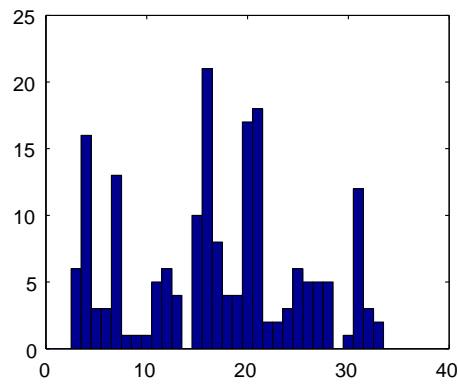

Industrial sector classification

Figure 2. Summary of the Data

sure in our model correspond to the instantaneous interest rate in the interest rate term structure models. There is no discrimination between the state variables in the interest rate models. But in our model, we should discriminate the state variables by intrinsic to each individual entity or common to whole entities. We assume that there exists some real valued stochastic process named Credit Quality Indicator (CQI) for every issuer which represents its credit reliabilities, and some real valued stochastic processes named Macro Credit Indicators (MCIs) which are common to whole entities, and the loss-adjusted hazard rates are the function of CQI and MCIs.

In our model, the yield term structure is defined for every CQI values. So it is extended to the Yield Spread Term-Quality Surface (YSTQS) which is defined on the space of term and credit reliability of the issuer, and express their yield spread from the default free yield.

Under the some technical assumptions and no arbitrage principle, we show that the yield spreads of discount corporate bonds are represented by some function of issuer's CQI, MCIs, and its duration. And we derive 
the partial differential equation which the function should hold. Solving this PDE, we get the YSTQS for each value of MCIs. Therefore MCIs have roles to fluctuate the YSTQS dynamically. This means that the yield spread term structure for some specified CQI will change depend to the current value of MCIs.

More over, we introduce the dynamic mapping function which transrate credit ratings to CQIs. We show its estimated result in advance (figure 3). Remember that the distribution of credit ratings is stable, regardless of the change of yield spreads. This function expresses the averaged change of credit reliability which is not captured by the migration of credit ratings.

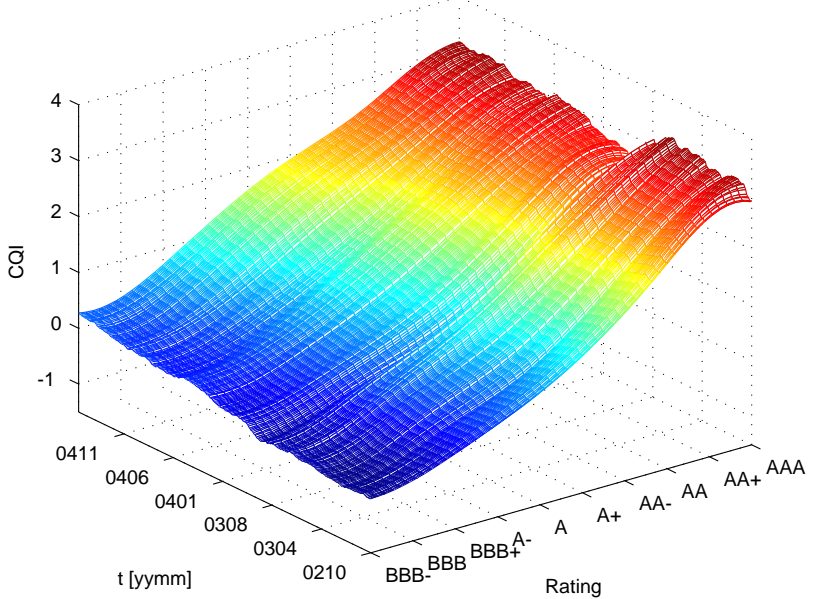

Figure 3. Mapping function which translates Credit Ratings to CQIs at each time point.

There exist researches which proposed similar model of ours. Douady and Jeanblanc (2002) derived no-arbitrage condition of the dynamics of 'YSTQS'. Their 'rating' and 'spread field' correspond to 'CQI' and 'YSTQS' in our model respectively. In their model, entities will default at some specified point of 'rating'. They modeled the dynamics of 'spread field' like Heath, Jarrow and Morton. But they do not investigate their model empirically. Bielecki, Crépey, Jeanblanc and Rutkowski (2005) discussed a general class of markov chain model modulated by Levy-like process. Their main aim is to price the derivatives of multi-default, which are basket default swaps, CDOs, and so on. The model of Feldhütter and Lando (2005) is constructed by (discrete) credit rating of individual obligor and 6 of common factors which drive risk free interest rate, credit spread and specific spread for swap rates and treasuries. They derived yield term structure for each credit class 
as function of state variables, and estimated the parameters of their model empirically.

The major difference of our model from those researches is that we characterized individual obligor by diffusion processes, Credit Quality Indicators which are not directly related to the credit rating or default, but they drive default hazard rates. Because our aim is to express the yield spread of defaultable bonds systematically and we do not sure that they are determined by credit ratings.

We demonstrate an empirical analysis of our model based on daily data of yield spreads, credit ratings, and durations of corporate bonds in the market. We estimate the model parameters and paths of the MCIs and CQIs in a context of filtering by pseudo-likelihood, with observation models, which relate the MCIs and CQIs to the yield spread, and the CQIs to the credit ratings.

The rest of this article is organized as follows. We formulate our model in section 2, define the interest rate, hazard rates, MCIs, CQIs, and others, and show the representation of risk free and corporate bond prices by them. Furthermore, we show an example of our model. In section 3, we estimate the model parameters and paths of the MCIs and CQIs by empirical analysis. Section 4 concludes this article.

\section{The Model}

\subsection{REVIEW OF the PRICING THEOREM OF DEFAUltable BONDS}

Let us review the pricing theorem of defaultable bonds based on external hazard rates briefly.

We consider a frictionless market, where default free discount bonds, discount corporate bonds issued by $N$ entities, and default free saving account are traded. We assume that their prices are described on a complete probability space, $(\Omega, \mathcal{F}, P)$.

Fix the time horizon $\bar{T}$. Denote the default time of entities by a set of random times $\tau_{i}: \Omega \rightarrow[0, \bar{T}] \cup\{\infty\}, i=1, \ldots, N$, and the default indicator of them by $N_{t}^{i}:=1_{\tau_{i} \leq t}$, which describe the state of their default at time $t$.

Denote the risk free instantaneous interest rate by $r .: \Omega \times[0, \bar{T}] \rightarrow$ $R^{++}$, and the price process of saving account as $S_{t}:=\exp \left(\int_{0}^{t} d s r_{s}\right)$. Moreover, denote the price process of unit principal default free discount bond $^{1}$ with maturity $T \in[0, \bar{T}]$ by $p_{\cdot, T}: \Omega \times[0, T] \rightarrow[0,1]$, and

\footnotetext{
${ }^{1}$ In most case, corporate bonds are coupon bonds. And it is reasonable to assume that the treatment of principals and coupons are different at the recovery. So the properness to evaluate coupon bonds as the sum of discount bonds is doubtful in
} 
the price process of unit principal corporate discount bond with maturity $T \in[0, \bar{T}]$ issued by corporate $i=1, \ldots, N$ by $p_{\cdot, T}^{i}: \Omega \times[0, T] \rightarrow$ $[0,1]$. We assume that $E^{P}\left[S_{\bar{T}}\right]<\infty$, and both of $p_{t, T}$ and $p_{t \wedge \tau_{i}-, T}^{i}$ are right continuous and left limit processes ${ }^{2}$.

Investors of unit principal default free discount bond will receive 1 at its maturity. Besides the investor of unit principal discount corporate bond will receive 1 at its maturity $T$ in the non-default case $\left(\tau_{i}>T\right)$, and receive $\left(1-L_{i}\right) p_{\tau_{i}-, T}^{i}$ at its default time $\tau_{i}$ in the default case $\left(\tau_{i} \leq T\right)$, where $L_{i}: \Omega \rightarrow(0,1]$ represents the fractional loss rate of market value. Note that we do not assume that $\tau_{i}$ and $L_{i}$ are mutually independent. Define the recovery process $R_{\cdot, T}^{i}: \Omega \times[0, T] \rightarrow[0,1)$ as,

$$
R_{t, T}^{i}:=N_{t}^{i}\left(1-L_{i}\right) p_{\left(t \wedge \tau_{i}\right)-, T}^{i} .
$$

In summary, at $t \in[0, T]$, the defaultable bonds' prices are;

$$
\begin{array}{llll}
p_{t, T}^{i}>0 & \text { if } t<\tau_{i}, & p_{t, T}^{i}=0 & \text { if } \tau_{i}<t \\
p_{T, T}^{i}=1 & \text { if } \tau_{i}>T, & p_{\tau_{i}, T}^{i}=R_{\tau_{i}, T}^{i} & \text { if } \tau_{i} \leq T .
\end{array}
$$

Define a filtration $\left\{\mathcal{F}_{t}^{M}\right\}_{t \in[0, \bar{T}]}$ which represents the information of all agents in the market up to time $t$ as follows. ${ }^{3}$

$$
\begin{aligned}
\mathcal{F}_{t}^{M}:= & \bigvee_{T \in[0, \bar{T}]}\left(\sigma\left\{p_{s, T} ; s \leq t \wedge T\right\} \vee \bigvee_{i=1, \ldots, N} \sigma\left\{p_{s, T}^{i} ; s \leq t \wedge T\right\}\right) \\
& \vee \sigma\left\{r_{s} ; s \leq t\right\} \vee \bigvee_{i=1}^{N}\left(\mathcal{D}_{t}^{i} \vee \mathcal{R}_{t}^{i}\right), \\
\mathcal{D}_{t}^{i}:= & \sigma\left\{N_{s}^{i} ; s \leq t\right\}, \quad \mathcal{R}_{t}^{i}:=\bigvee_{T \in[0, \bar{T}]} \sigma\left\{R_{s, T}^{i} ; s \leq t \wedge T\right\} .
\end{aligned}
$$

We call it the Market Filtration and assume that $\mathcal{F}_{\bar{T}}^{M} \subseteq \mathcal{F}$. Note that the definition of $\mathcal{R}_{t}^{i}$ means that after the default of the $i^{\text {th }}$ entity, the recovery value of the corporate bonds issued by it which have not maturated at $\tau_{i}$ is revealed immediately. Conversely, if the issuer has not defaulted until the bond maturity, its recovery value is never revealed to the market.

Require that the market is no-arbitrage, i.e. there exists at least one martingale measure $Q$ equivalent to $P$ under which $p_{t, T} S_{t}^{-1}$ and

$\overline{\text { practice. }}$ But in this paper, we only consider the evaluation of discount bond to avoid the argument to be too complicated.

${ }^{2}$ For any stochastic process $X(t, \omega)$, denote $\Delta X:=X(t, \omega)-X(t-, \omega)$ and let $X(0-, \omega)=0, \forall \omega \in \Omega$.

3 We assume that the all filtrations are complete and right continuous. 
$p_{t, T}^{i} S_{t}^{-1}$ become $\left(Q, \mathcal{F}_{t}^{M}\right)$-martingales at $t \in[0, T]$ and $t \in\left[0, T \wedge \tau_{i}\right]$ respectively ${ }^{4}$.

Hence $N_{t}^{i}$ is a submartingale, by Doob-Meyer's theorem ${ }^{5}$, there exists a $\left(\mathcal{F}_{t}^{M}\right)$-predictable right continuous nonnegative monotonically nondecreasing process $\tilde{\Lambda}_{t}^{i}$, where $\tilde{\Lambda}_{0}^{i}=0$ for every $i=1, \ldots, N$, and $N_{t}^{i}-\tilde{\Lambda}_{t}^{i}$ is a $\left(Q,\left(\mathcal{F}_{t}^{M}\right)\right)$-martingale. $\tilde{\Lambda}_{t}^{i}$ is called $\left(Q,\left(\mathcal{F}_{t}^{M}\right)\right)$-cumulative hazard process of the $i^{\text {th }}$ entity. ${ }^{6}$

ASSUMPTION 1. $\tilde{\Lambda}_{t}^{i}$ is absolutely continuous and by some $\left(\mathcal{F}_{t}^{M}\right)$ progressively measurable process $\tilde{h}_{t}^{i}>0$, described as,

$$
\tilde{\Lambda}_{t}^{i}=\int_{0}^{t}\left(1-N_{s-}^{i}\right) \tilde{h}_{s}^{i} d s
$$

And it is bounded as $E^{Q}\left[e^{\tilde{\Lambda}_{T}^{i}} \mid \tau_{i} \geq T\right]<\infty$.

$\tilde{h}_{t}^{i}$ is called $\left(Q,\left(\mathcal{F}_{t}^{M}\right)\right)$-hazard rate process of the $i^{\text {th }}$ entity. This assumption implies that $\tau_{i}$ is a totally inaccessible stopping time.

PROPOSITION 2. If the assumption 1 hold, for any $i=1, \ldots, N, T \in$ $[0, \bar{T}]$, there exists a $\left(\mathcal{F}_{t}^{M}\right)$-progressively measurable process $\gamma_{t}^{i} \in\left[0, \tilde{h}_{t}^{i}\right]$ with which the cumulative loss-adjusted hazard process $A_{t}^{i}$ is defined as,

$$
A_{t}^{i}=\int_{0}^{t} d s\left(1-N_{s}^{i}\right) \gamma_{s}^{i}
$$

and $N_{t}^{i} L_{i}-A_{t}^{i}$ becomes a $\left(Q,\left(\mathcal{F}_{t}^{M}\right)\right)$-martingale.

We call $\gamma^{i}$ the $\left(Q,\left(\mathcal{F}_{t}^{M}\right)\right)$-loss-adjusted hazard rate process of the $i^{\text {th }}$ entity.

THEOREM 3. For any $\left(Q,\left(\mathcal{F}_{t}^{M}\right)\right)$-semimartingale $X_{{ }_{\cdot} T}^{i}: \Omega \times[0, T] \rightarrow$ $R$, the following two conditions are equivalent;

(i) The defaultable bond prices are represented by such $X_{{ }_{\cdot} T}^{i}$ as,

$$
p_{t, T}^{i}=\left(1-N_{t}^{i}\right) X_{t, T}^{i} e^{\int_{0}^{t} r_{s}+\gamma_{s}^{i} d s}+\left(1-N_{t-}^{i}\right) R_{t, T}^{i} .
$$

\footnotetext{
${ }^{4}$ We do not assume that the completeness of the market, so the uniqueness of the risk neutral measure is not guaranteed. On the rest of this article, we only state that if our assumptions hold in some risk neutral measure, we can get the results.

5 See Protter (2003) III-3.

6 See Blanchet-Scallied and Jeanblanc (2004) or Bielecki and Rutkowski (2001) Chapter $5 \sim 8$ for detail of mathematical formulation about the intensity based models.
} 
(ii) $\hat{M}_{\cdot, T}^{i}: \Omega \times[0, T] \rightarrow R$ which is defined by such $X_{\cdot, T}^{i}$ as,

$$
\hat{M}_{t, T}^{i}:=\left(1-N_{t}^{i}\right) X_{t, T}^{i}+N_{t}^{i} X_{\tau_{i}-, T}^{i},
$$

is a $\left(Q,\left(\mathcal{F}_{t}^{M}\right)\right)$-martingale, and it satisfies the terminal condition,

$$
\left(1-N_{T}^{i}\right) X_{T, T}^{i}=\left(1-N_{T}^{i}\right) e^{-\int_{0}^{T} r_{s}+\gamma_{s}^{i} d s} .
$$

This theorem states that the only $\left(Q,\left(\mathcal{F}_{t}^{M}\right)\right)$-loss adjusted hazard rate processes are important to price defaultable bonds ${ }^{7}$.

\subsection{The Micro-Macro Indicators}

We introduce state vectors $x_{t}: \Omega \times[0, \bar{T}] \rightarrow \mathcal{X} \subseteq R^{M}$ and $z_{t}: \Omega \times$ $[0, \bar{T}] \rightarrow \mathcal{Z}^{N} \subseteq R^{N}$.

We put the following assumptions on the risk free interest rate and the loss-adjusted hazard rates.

ASSUMPTION 4. The $\left(Q,\left(\mathcal{F}_{t}^{M}\right)\right)$-loss-adjusted hazard rate process at time $t \in[0, \bar{T}]$ of the $i^{\text {th }}$ entity, $\gamma_{t}^{i}$, is represented by a function of $x_{t}$ and $z_{t}^{i}$, where, $z_{t}^{i}$ denotes the $i^{\text {th }}$ component of $z_{t} .^{8}$

$$
\gamma_{t}^{i}=\gamma\left(x_{t}, z_{t}^{i}\right), \quad \gamma \in C^{2,2}\left(\mathcal{X} \times \mathcal{Z} \rightarrow R^{++}\right) .
$$

ASSUMPTION 5. The risk free instantaneous interest rate at time $t \in[0, \bar{T}]$ is represented by a function of $x_{t}$ and a $\left(\mathcal{F}_{t}^{M}\right)$-mesurable stochastic process $g .: \Omega \times[0, \bar{T}] \rightarrow R^{+}$, which is independent of state variables.

$$
r_{t}=r\left(x_{t}\right)+g_{t}, \quad r \in C^{2}\left(\mathcal{X} \rightarrow R^{+}\right) .
$$

We call each component of $x_{t}$ Macro Credit Indicator (MCI), hence they affect default free interest rate and default intensities of all entities. Conversely, we call $z_{t}^{i}$ Credit Quality Indicator (CQI) of the $i^{\text {th }}$ entity, hence it only affects the hazard rate of the $i^{\text {th }}$ entity. The macro

\footnotetext{
7 This result is similar to the Theorem 1 in Duffie and Singleton (1999), but it holds in more general case.

Kusuoka (1999) states that it needs some technical assumptions to derive the standard model of credit risk.

Without such additional assumptions, we can not represent defaultable bond prices by expectation form anymore. But we can still derive partial differential equations or execute Monte Carlo simulation about bond prices based on our theorem.

${ }^{8}$ When $t \geq \tau_{i}, \gamma_{t}^{i}$ has no meaning in finance.
} 
credit indicator represents the economic state. And the credit quality indicator represents the credit reliability of individual entity under the condition of the macro credit indicator.

Define the following filtrations at $t \in[0, \bar{T}]$.

$$
\begin{aligned}
\mathcal{G}_{t} & :=\sigma\left\{x_{s}, z_{s} ; s \leq t\right\}, \\
\mathcal{F}_{t} & :=\mathcal{G}_{t} \vee \bigvee_{i=1}^{N} \mathcal{D}_{t}^{i} \vee \mathcal{R}_{t}^{i} \vee \sigma\left\{g_{s} ; s \leq t\right\} .
\end{aligned}
$$

$\tau_{i}$ are $\left(\mathcal{F}_{t}\right)$-stopping time. Let all the component of $x_{t}$ and $z_{t}$ be $\left(\mathcal{G}_{t}\right)$ semimartingales.

ASSUMPTION 6. We assume $\mathcal{G}_{t} \subseteq \mathcal{F}_{t}^{M}, \forall t \in[0, \bar{T}]$, i.e., $x_{t}$ and $z_{t}$ are $\left(\mathcal{F}_{t}^{M}\right)$-measurable.

Therefore trivially, $\mathcal{F}_{t} \subseteq \mathcal{F}_{t}^{M}, \forall t \in[0, \bar{T}]$.

We restrict our discussion within the case of that the state vectors are some Markov diffusion processes. Let $M$ and $N$ dimensional mutually independent $P$-standard Brownian motions $w_{t}^{x}, w_{t}^{z}, t \in[0, \bar{T}]$ be described on $(\Omega, \mathcal{F}, P)$ respectively, and define,

$$
d B_{t}^{i}:=\sqrt{1-\rho_{x z}^{2}} v_{i} d w_{t}^{z}+\rho_{x z} d w_{t}^{x},
$$

where $\rho_{x z}$ is a constant $M$-dimensional (row) vector with $\rho_{x z}^{2} \in[0,1$ ) and $v_{i}, i=1, \ldots, N$ are mutually linear independent $N$-dimensional (row) vectors with $\left|v_{i}\right|=1$.

Let the state vectors $x_{t}$ and $z_{t}$ be the solutions of following simultaneous stochastic differential equations respectively.

$$
\begin{aligned}
d x_{t} & =\mu_{x}\left(x_{t}\right) d t+\sigma_{x}\left(x_{t}\right) d w_{t}^{x}, \\
d z_{t}^{i} & =\mu_{z}\left(x_{t}, z_{t}^{i}\right) d t+\sigma_{z}\left(x_{t}, z_{t}^{i}\right) d B_{t}^{i} .
\end{aligned}
$$

The each coefficient function must satisfy the conditions that the equations have strong solutions.

Hence the martingale representation theorem, Radon-Nikodým density process, $\eta_{t}$, between $P$ and $Q$ under $\mathcal{G}_{t}$ is given by some predictable $\left(\mathcal{G}_{t}\right)$-progressively measurable process, $\left(\beta^{x}, \beta^{z}\right): \Omega \times[0, \bar{T}] \rightarrow R^{M+N}$ as follows.

$$
\eta_{t}:=\left.\frac{d Q}{d P}\right|_{\mathcal{G}_{t}}=1+\int_{0+}^{t} \eta_{s-}\left(\beta_{s}^{x} d w_{s}^{x}+\beta_{s}^{z} d w_{s}^{z}\right) .
$$

Define

$$
\beta_{t}^{i}:=\sqrt{1-\rho_{x z}^{2}} v_{i} \beta_{t}^{z}+\rho_{x z} \beta_{t}^{x} .
$$


ASSUMPTION 7. $\beta_{t}^{x}, \beta_{t}^{i}, \forall t \in[0, \bar{T}], \forall i=1, \ldots, N$ are represented by,

$$
\beta_{t}^{x}=\beta^{x}\left(x_{t}\right), \quad \beta_{t}^{i}=\beta^{i}\left(x_{t}, z_{t}^{i}\right) .
$$

Define $\tilde{w}_{t}^{x}$ and $\tilde{B}_{t}^{i}, i=1, \ldots, N$ as,

$$
\begin{aligned}
d \tilde{w}_{t}^{x} & =d w_{t}^{x}+\beta_{t}^{x} d t, \\
d \tilde{B}_{t}^{i} & =d B_{t}^{i}+\beta_{t}^{i} d t .
\end{aligned}
$$

From the Girsanov theorem, they are $Q$-standard Brownian motions. Equation (5) can be rewritten as,

$$
\begin{aligned}
d x_{t} & =\tilde{\mu}_{x}\left(x_{t}\right) d t+\sigma_{x}\left(x_{t}\right) d \tilde{w}_{t}^{x}, \\
d z_{t}^{i} & =\tilde{\mu}_{z}\left(x_{t}, z_{t}^{i}\right) d t+\sigma_{z}\left(x_{t}, z_{t}^{i}\right) d \tilde{B}_{t}^{i},
\end{aligned}
$$

where,

$$
\begin{aligned}
\tilde{\mu}_{x}\left(x_{t}\right) & :=\mu_{x}\left(x_{t}\right)-\sigma_{x}\left(x_{t}\right) \beta_{t}^{x}\left(x_{t}\right), \\
\tilde{\mu}_{z}\left(x_{t}, z_{t}^{i}\right) & :=\mu_{z}\left(x_{t}, z_{t}^{i}\right)-\sigma_{z}\left(x_{t}, z_{t}^{i}\right) \beta^{i}\left(x_{t}, z_{t}^{i}\right) .
\end{aligned}
$$

Let us consider the default free bond prices. We assume that there exists the following function.

ASSUMPTION 8. Let $f \in C^{2,1}\left(\mathcal{X} \times[0, \bar{T}] \rightarrow R^{+}\right)$be a solution of the following partial differential equation, and assume its existence ${ }^{9}$.

$$
\begin{aligned}
\tilde{\mu}_{x}^{T}(x) \partial_{x} f(x, \tau)+\frac{1}{2} \operatorname{Tr}\left[\sigma _ { x } ( x ) \sigma _ { x } ^ { T } ( x ) \left(\partial_{x}^{T} \partial_{x} f(x, \tau)\right.\right. \\
\left.\left.\quad-\left(\partial_{x}^{T} f(x, \tau)\right)\left(\partial_{x} f(x, \tau)\right)\right)\right]-\partial_{\tau} f(x, \tau)+r(x)=0 .
\end{aligned}
$$

And its terminal condition is given as follows.

$$
f(x, 0)=0, \quad \forall x \in \mathcal{X} .
$$

Then, the following proposition should hold.

PROPOSITION 9. At any $t \in[0, T]$, the price of unit principal default free discount bond with any maturity $T \in[0, \bar{T}]$ is represented by $f(\cdot, \cdot)$ as,

$$
p_{t, T}=\exp \left(-f\left(x_{t}, T-t\right)\right) E^{Q}\left[\exp \left(-\int_{t}^{T} g_{s} d s\right) \mid \mathcal{F}_{t}^{M}\right] .
$$

\footnotetext{
${ }^{9} a^{T}$ denotes the transposed matrix of $a$. The operator $\partial_{x}$ and $\partial_{x}{ }^{T}$ generate $M$ dimensional column and row vectors respectively. $\operatorname{Tr}[\cdot]$ denotes trace of matrices.
} 
Proof. Hence $Q$ is a martingale measure, the price of default free discount bonds are represented as follows,

$$
p_{t, T}=M_{t, T} S_{t}, \quad M_{t, T}:=E^{Q}\left[S_{T}^{-1} \mid \mathcal{F}_{t}^{M}\right] .
$$

We should show,

$$
\begin{aligned}
M_{t, T} & =S_{t}^{-1} \exp \left(-f\left(x_{t}, T-t\right)\right) E^{Q}\left[\exp \left(-\int_{t}^{T} g_{s} d s\right) \mid \mathcal{F}_{t}^{M}\right] \\
& =\exp \left(-f\left(x_{t}, T-t\right)-\int_{0}^{t} r\left(x_{s}\right) d s\right) E^{Q}\left[\exp \left(-\int_{0}^{T} g_{s} d s\right) \mid \mathcal{F}_{t}^{M}\right] .
\end{aligned}
$$

Obviously, it holds the terminal condition that $M_{T, T}=S_{T}^{-1}$. Hence, $x$. and $g$. are mutually independent, the problem is only to show that the part of $x_{t}$ is a martingale. And it is easily checked by the Ito formula.

Q.E.D.

Next, we consider the corporate bonds, which are exposed to the default risk. We assume that there exists the following function.

ASSUMPTION 10. Let $Y \in C^{2,2,1}\left(\mathcal{X} \times \mathcal{Z} \times[0, \bar{T}] \rightarrow R^{+}\right)$, be a solution of the following partial differential equation, and assume its existence.

$$
\begin{aligned}
& \tilde{\mu}_{x}^{T}(x) \partial_{x} Y(x, z, \tau)+\tilde{\mu}_{z}(x, z) \partial_{z} Y(x, z, \tau) \\
& +\frac{1}{2} \operatorname{Tr}\left[\sigma _ { x } ( x ) \sigma _ { x } ^ { T } ( x ) \left(\partial_{x}^{T} \partial_{x} Y(x, z, \tau)-\left(\partial_{x}^{T} Y(x, z, \tau)\right)\left(\partial_{x} Y(x, z, \tau)\right)\right.\right. \\
& \left.\left.\quad-2\left(\partial_{x}^{T} Y(x, z, \tau)\right)\left(\partial_{x} f(x, \tau)\right)\right)\right] \\
& +\frac{1}{2} \sigma_{z}^{2}(x, z)\left(\partial_{z}^{2} Y(x, z, \tau)-\left(\partial_{z} Y(x, z, \tau)\right)^{2}\right) \\
& +\rho_{x z} \sigma_{z}(x, z) \sigma_{x}^{T}(x)\left(\partial_{x} \partial_{z} Y(x, z, \tau)-\left(\partial_{x} f(x, \tau)+\partial_{x} Y(x, z, \tau)\right)\right. \\
& \left.\left(\partial_{z} Y(x, z, \tau)\right)\right)-\partial_{\tau} Y(x, z, \tau)+\gamma(x, z)=0 .
\end{aligned}
$$

And its terminal condition is given as follows.

$$
\forall x \in \mathcal{X}, z \in \mathcal{Z}, \quad Y(x, z, 0)=0 .
$$

Then the following theorem should hold.

THEOREM 11. The market price of the unit principal discount corporate bond with any maturity $T \in[0, \bar{T}]$ issued by arbitrary entity $i$ is represented as follows at any $t \in[0, T]$.

$$
p_{t, T}^{i}=\left(1-N_{t}^{i}\right) p_{t, T} \exp \left(-Y\left(x_{t}, z_{t}^{i}, T-t\right)\right)+\left(1-N_{t-}^{i}\right) R_{t, T}^{i} .
$$


Proof. Put,

$$
X_{t, T}^{i}=p_{t, T} \exp \left(-Y\left(x_{t}, z_{t}^{i}, T-t\right)\right) e^{-\int_{0}^{t} r_{s}+\gamma_{s}^{i} d s}
$$

and check it satisfies the condition of theorem 3 .

Q.E.D.

Finally, it has shown that $p_{t, T}$ and $p_{t, T}^{i}$ are $\mathcal{F}_{t}$-measurable. So we have the following remark.

REMARK 12. If the all assumptions are satisfied, the market filtration $\mathcal{F}_{t}^{M}$ is equivalent to the filtration $\mathcal{F}_{t}$, i.e. $\mathcal{F}_{t}^{M}=\mathcal{F}_{t}, \forall t \in[0, \bar{T}]$.

Now we can check weather the assumption 6 of $\mathcal{G}_{t} \subseteq \mathcal{F}_{t}^{M}$ is consistent with other assumptions or not. To hold it, it required that there is no redundant risk with $x_{t}$ or $z_{t}$. One of the sufficient conditions is given as follows.

$$
\begin{array}{ll}
\left|\partial_{x}^{i} r(x)\right|+\left|\partial_{x}^{i} \gamma(x, z)\right| \neq 0, & \left|\partial_{z} \gamma(x, z)\right| \neq 0, \\
\left|\sigma_{x}(x)\right| \neq 0, & \left|\sigma_{z}(x, z)\right| \neq 0, \quad \text { a.e. }
\end{array}
$$

DEFINITION 13. The Yield Spread Term-Quality Surface at time $t$ is defined as,

$$
Y S T Q S_{t}(z, T)=\frac{1}{T} \ln \left(\frac{p_{t, t+T}^{i}}{p_{t, t+T}}\right)_{z_{t}^{i}=z, \tau_{i}>t}=\frac{Y\left(x_{t}, z, T\right)}{T} .
$$

\subsection{An Example of Our Model}

\section{Model}

Assume that there is only one component of the MCI and the MCI does not affect interest rate, i.e., $r(\cdot)=0$, so we can drop $f(\cdot, \cdot)$ from equation (9). We further assume that this scalar MCI is a square root process, i.e. the coefficient functions of equation (6) are given as follows,

$$
\begin{aligned}
d x_{t} & =\kappa_{x}\left(\theta_{x}-x_{t}\right) d t+\sigma_{x} \sqrt{x_{t}} d w_{t}^{x} \\
& =\kappa_{x}\left(\tilde{\theta}_{x}-x_{t}\right) d t+\sigma_{x} \sqrt{x_{t}} d \tilde{w}_{t}^{x},
\end{aligned}
$$

where $\sigma_{x}>0, \kappa_{x}>0, \tilde{\theta}_{x}>0, \theta_{x}>0$. The square root process is popular in finance as an example of stable positive definite processes like instantaneous interest rates or hazard rates. 
CQI Model

We put CQI $z_{t}$ as $\mathrm{OU}$ processes, i.e.,

$$
\begin{aligned}
d z_{t}^{i} & =\kappa_{z}\left(\theta_{z}-z_{t}^{i}\right) d t+\sqrt{2 \kappa_{z}} d B_{i}(t) \\
& =\kappa_{z}\left(\tilde{\theta}_{z}-z_{t}^{i}\right) d t+\sqrt{2 \kappa_{z}} d \tilde{B}_{i}(t),
\end{aligned}
$$

where $\kappa_{z}>0$. The unconditional mean and variance of this process are $\theta_{z},\left(\tilde{\theta}_{z}\right.$ on measure $\left.Q\right)$ and 1 at any $t$ respectively. We consider this stability is suitable for the CQI.

And put the loss-adjusted hazard rate function as follows,

$$
\gamma(x, z):=\frac{\bar{\gamma} x}{2 x_{0}}(\tanh (\phi(z))+1),
$$

where $\phi(\cdot)$ is a cubic spline function. We fix $\gamma\left(x_{0}, z_{0}\right)=\gamma_{0}$ to normalize $x_{t} \cdot \gamma(\cdot, \cdot)$ satisfies the upper and lower restrictions $\gamma(x, z) \in\left(0, \bar{\gamma} x / x_{0}\right)$ in $z \in R$ in spite of its high flexibility. So we can avoid the explosion of the the hazard rate.

To solve the PDE (9) by finite differential method, we put the terminal condition of $Y(\cdot, \cdot, \cdot)$ as follows,

$$
\begin{gathered}
Y(x, z, 0)=0, \\
\partial_{z}^{2} Y(x, z, \tau)=0, \text { for }|z| \rightarrow \infty, \\
\partial_{x}^{2} Y(x, z, \tau)=0, \text { for } x \rightarrow \infty .
\end{gathered}
$$

\section{Data Analysis}

We evaluate the model parameters and paths of MCI and CQIs for all entities from daily yield spreads, durations, and credit ratings of corporate bonds. The YSTQS of each time point will be revealed as the result. We use the coupon bond data, but to reduce the calculation cost, we evaluate them as discount bonds those have same yield and duration with the original coupon bonds.

Though the model is nonlinear, we apply linear approximations at each time step. The observation noise and the conditional distribution of state variables are approximated by multidimensional normal distribution, and apply the maximum pseudo-likelihood estimation.

\subsection{The Observation Model}

Denote the set of yield spreads, durations, and credit ratings of corporate bonds by $\left\{\left(\tilde{Y}_{t}^{i, n}, \tau_{t}^{i, n}, \tilde{\xi}_{t}^{i}\right): t \in \mathcal{T}, n \in \mathcal{N}_{t}^{i}, i \in \mathcal{I}_{t}\right\}$, where the super script $i$ denotes their issuer, $n$ identifies the issue, and the sub 
script $t$ denotes the calender time respectively. $\mathcal{I}_{t}$ represents the set of entities of which data exists at the time point $t \in \mathcal{T}=\{1, \ldots, T\}$, and $\mathcal{N}_{t}^{i}$ represents the set of bonds issued by $i$ of which data exists at the time point $t . \tilde{\xi}_{t}^{i} \in \Xi$ are the observed credit ratings from AAA to BBB-, where $\Xi:=\left\{\zeta_{\mathrm{AAA}}, \ldots, \zeta_{\mathrm{BBB}}\right\} \subseteq R$.

Denote $\tilde{Y}_{t}^{*}:=\left\{\tilde{Y}_{t}^{i, n} \mid n \in \mathcal{N}_{t}^{i}, i \in \mathcal{I}_{t}\right\}, \tilde{\xi}_{t}:=\left\{\tilde{\xi}_{t}^{i} \mid i \in \mathcal{I}_{t}\right\}, \tilde{Y}_{\{t\}}:=$ $\left\{\tilde{Y}_{s}: s \leq t\right\}, \tilde{\xi}_{\{t\}}:=\left\{\tilde{\xi}_{s}: s \leq t\right\} . \tilde{Y}_{t}$ and $\tilde{\xi}_{t}$ are the set of information about the yield spreads and credit ratings observed at time point $t$ respectively, and $\tilde{Y}_{\{t\}}$ and $\tilde{\xi}_{\{t\}}$ are those of observed up to time $t$ respectively.

We use the following observation models.

\section{Observation model of the yield spreads:}

$$
\begin{aligned}
& \tilde{Y}_{t}^{i, n}=Y\left(x_{t}, z_{t}^{i}+\epsilon_{z, t}^{i, n}, \tau_{t}^{i, n}\right)+\tau_{t}^{i, n}\left(\delta_{l}+\epsilon_{y, t}^{i, n}\right), \\
& \epsilon_{z, t}^{i, n} \sim N\left(0, \lambda_{z}^{2}\right), \quad \epsilon_{y, t}^{i, n} \sim N\left(0, \lambda_{y}^{2}\right) .
\end{aligned}
$$

\section{Observation model of the credit ratings:}

$$
\begin{gathered}
P\left(\tilde{\xi}_{t}^{i} \mid z_{t}^{i}\right)=\frac{1}{A_{z}\left(z_{t}^{i}, t\right)} \exp \left(-\frac{\left(z_{t}^{i}-\psi\left(\tilde{\xi}_{t}^{i}, t\right)\right)^{2}}{2 \lambda_{\xi}\left(\tilde{\xi}_{t}^{i}, t\right)^{2}}\right), \\
A_{z}(z, t):=\sum_{\xi \in \Xi} \exp \left(-\frac{(z-\psi(\xi, t))^{2}}{2 \lambda_{\xi}(\xi, t)^{2}}\right) .
\end{gathered}
$$

See the observation model of the yield spreads. We considered that there exist two kinds of noise source in the observation of yield spreads. The first is misestimation of CQI, which is represented by $\epsilon_{z, t}^{i, n}$. The second is simple noise on the yield spreads, which is represented by $\epsilon_{y, t}^{i, n}$. Meanwhile, $\delta_{l}>0$ is the liquidity adjustment parameter. We assume that the noises are mutually independent in $i, n$ and $t^{10}$.

On the other hand, the observation model of the credit ratings gives obserbation probability of credit ratings. We transform the credit ratings to CQIs with the time depending mapping function $\psi: \Xi \times \mathcal{T} \rightarrow \mathcal{Z}$. We denote $\tilde{z}_{t}^{i}:=\psi\left(\tilde{\xi}_{t}^{i}, t\right)$. The magnitude of CQIs observation error estimated from the credit ratings are denoted by $\lambda_{\xi}: \Xi \times \mathcal{T} \rightarrow R^{+}$. $A_{z}: \mathcal{Z} \times \mathcal{T} \rightarrow R^{++}$is the normalize coefficient.

\footnotetext{
10 There is another choice that $\epsilon_{z, t}^{i, n}$ does not depend to $n$. But it makes the correlation matrix non diagonal.
} 
Let us derive the recurrence formula of likelihood from above observation models. At first, decompose the likelihood function to the initial probability function and the conditional probability functions at each time point as follows.

$$
\begin{aligned}
P\left(\tilde{Y}_{\{T\}}, \tilde{\xi}_{\{T\}}\right) & =P\left(\tilde{Y}_{T}, \tilde{\xi}_{T} \mid \tilde{Y}_{\{T-1\}}, \tilde{\xi}_{\{T-1\}}\right) P\left(\tilde{Y}_{\{T-1\}}, \tilde{\xi}_{\{T-1\}}\right) \\
& =\left(\prod_{t=1}^{T} P\left(\tilde{Y}_{t}, \tilde{\xi}_{t} \mid \tilde{Y}_{\{t-1\}}, \tilde{\xi}_{\{t-1\}}\right)\right) P\left(\tilde{Y}_{\{0\}}, \tilde{\xi}_{\{0\}}\right) .
\end{aligned}
$$

The probability function of the observations at each time point and the filtered probability function of the state variables are represented as follows respectively.

$$
\begin{aligned}
& P\left(\tilde{Y}_{t}, \tilde{\xi}_{t} \mid \tilde{Y}_{\{t-1\}}, \tilde{\xi}_{\{t-1\}}\right) \\
& \quad=\int P\left(x_{t} \in d x, z_{t} \in d z, \tilde{Y}_{t}, \tilde{\xi}_{t} \mid \tilde{Y}_{\{t-1\}}, \tilde{\xi}_{\{t-1\}}\right), \\
& P\left(x_{t}, z_{t} \mid \tilde{Y}_{\{t\}}^{\cdot}, \tilde{\xi}_{\{t\}}\right) \\
& \quad=\frac{P\left(x_{t}, z_{t}, \tilde{Y}_{t}, \tilde{\xi}_{\dot{t}} \mid \tilde{Y}_{\{t-1\}}, \tilde{\xi}_{\{t-1\}}\right)}{\int P\left(x_{t} \in d x, z_{t} \in d z, \tilde{Y}_{t}, \tilde{\xi}_{t} \mid \tilde{Y}_{\{t-1\}}, \tilde{\xi}_{\{t-1\}}\right),}
\end{aligned}
$$

where,

$$
P\left(x_{t}, z_{t}, \tilde{Y}_{t}^{\cdot}, \tilde{\xi}_{t} \mid \tilde{Y}_{\{t-1\}}, \tilde{\xi}_{\{t-1\}}\right)
$$

gives the conditional joint probability function of the state variables and the observations at each time point.

(filtering) The conditional joint probability function of the state variables and the observations at each time point are decomposed to the predicted probability function of the state variables and the probability function of the observations as follows.

$$
\begin{aligned}
P\left(x_{t}, z_{t}, \tilde{Y}_{t}, \tilde{\xi}_{t} \mid \tilde{Y}_{\{t-1\}}, \tilde{\xi}_{\{t-1\}}\right) & \\
= & \left(\prod_{i \in \mathcal{I}_{t}} \prod_{n \in \mathcal{N}_{t}^{i}} P\left(\tilde{Y}_{t}^{i, n} \mid x_{t}, z_{t}^{i}\right)\right)\left(\prod_{i \in \mathcal{I}_{t}} P\left(\tilde{\xi}_{t}^{i} \mid z_{t}^{i}\right)\right) \\
& P\left(x_{t}, z_{t} \mid \tilde{Y}_{\{t-1\}}^{\cdot}, \tilde{\xi}_{\{t-1\}}\right) .
\end{aligned}
$$

(prediction) The predicted probability function of the state variables are given by the filtered probability functions of the state variables at the previous time point and the transition probability of the state variables, which are determined by dynamics of the state variables as follows.

$P\left(x_{t}, z_{t} \mid \tilde{Y}_{\{t-1\}}^{\cdot}, \tilde{\xi}_{\{t-1\}}\right)$ 


$$
=\int P\left(x_{t} \mid x_{t-1}\right) P\left(z_{t} \mid x_{t-1}, z_{t-1}\right) P\left(x_{t-1} \in d x, z_{t-1} \in d z \mid \tilde{Y}_{\{t-1\}}, \tilde{\xi}_{\{t-1\}}\right) .
$$

We approximate the conditional joint probability function of MCI $x_{t}$ and CQIs $z_{t}$ by multidimensional normal distributions, and denote their means and covariance as follows.

$$
\begin{gathered}
\bar{x}_{t \mid s}:=E^{P}\left[x_{t} \mid \tilde{Y}_{\{s\}}, \tilde{\xi}_{\{s\}}\right], \quad V_{t \mid s}:=E^{P}\left[x_{t} x_{t}-\bar{x}_{t \mid s} \bar{x}_{t \mid s} \mid \tilde{Y}_{\{s\}}, \tilde{\xi}_{\{s\}}\right], \\
\bar{z}_{t \mid s}^{i}:=E^{P}\left[z_{t}^{i} \mid \tilde{Y}_{\{s\}}, \tilde{\xi}_{\{s\}}\right], \quad V_{t \mid s}^{i j}:=E^{P}\left[z_{t}^{i} z_{t}^{j}-\bar{z}_{t \mid s}^{i} \bar{z}_{t \mid s}^{j} \mid \tilde{Y}_{\{s\}}, \tilde{\xi}_{\{s\}}\right], \\
W_{t \mid s}^{i}:=E^{P}\left[x_{t} z_{t}^{i}-\bar{x}_{t \mid s} \bar{z}_{t \mid s}^{i} \mid \tilde{Y}_{\{s\}}, \tilde{\xi}_{\{s\}}\right] .
\end{gathered}
$$

We apply the following discrete approximation of state equation (5), where, $D$ denotes the time interval of the observations.

$$
\begin{aligned}
x_{t} \simeq & \left(\mu_{x}\left(\bar{x}_{t-1 \mid t-1}\right)-\frac{1}{2} \partial_{x} \mu_{x}\left(\bar{x}_{t-1 \mid t-1}\right) \bar{x}_{t-1 \mid t-1}\right) D \\
& +\left(1+\frac{1}{2} \partial_{x} \mu_{x}\left(\bar{x}_{t-1 \mid t-1}\right) D\right) x_{t-1}+\sigma_{x}\left(\bar{x}_{t-1 \mid t-1}\right) e_{t} \\
:= & M_{t-1}+K_{t-1} x_{t-1}+\varsigma_{t-1} e_{t}, \\
z_{t}^{i}= & \left(\mu_{z}\left(\bar{x}_{t-1 \mid t-1}, \bar{z}_{t-1 \mid t-1}^{i}\right)-\frac{1}{2} \partial_{z} \mu_{z}\left(\bar{x}_{t-1 \mid t-1}, \bar{z}_{t-1 \mid t-1}^{i}\right) \bar{z}_{t-1 \mid t-1}^{i}\right. \\
& \left.-\frac{1}{2} \partial_{x} \mu_{z}\left(\bar{x}_{t-1 \mid t-1}, \bar{z}_{t-1 \mid t-1}^{i}\right) \bar{x}_{t-1 \mid t-1}\right) D \\
& +\left(1+\frac{1}{2} \partial_{z} \mu_{z}\left(\bar{x}_{t-1 \mid t-1}, \bar{z}_{t-1 \mid t-1}^{i}\right) D\right) z_{t-1}^{i} \\
& +\frac{1}{2} \partial_{x} \mu_{z}\left(\bar{x}_{t-1 \mid t-1}, \bar{z}_{t-1 \mid t-1}^{i}\right) D x_{t-1}^{i}+\sigma_{z}\left(\bar{x}_{t-1 \mid t-1}, \bar{z}_{t-1 \mid t-1}^{i}\right) e_{t}^{i} \\
:= & M_{t-1}^{i}+L_{t-1}^{i} z_{t-1}^{i}+K_{t-1}^{i} x_{t-1}+\varsigma_{t-1}^{i} e_{t}^{i},
\end{aligned}
$$

where $e_{t}$ and $e_{t}^{i}$ are normally distributed stochastic variables with zero mean and following covariance, and independent of $e_{s}$ and $e_{s}^{i}$ if $t \neq s$.

$$
\begin{gathered}
\operatorname{Var}\left[e_{t}\right]=D, \quad \operatorname{Cov}\left[e_{t}, e_{t}^{i}\right]=\rho_{x z} D \\
\operatorname{Cov}\left[e_{t}^{i}, e_{t}^{j}\right]=\left(\left(1-\rho_{x z}^{2}\right) v_{i} v_{j}^{T}+\rho_{x z}^{2}\right) D:=\Omega^{i j} D .
\end{gathered}
$$

$\Omega^{i j}$ gives the correlation of fluctuation of CQI based on $G_{i}$ which denotes the industrial sector classification of the obligor $i$ as follows.

$$
\Omega^{i j}:=1_{i=j}+\rho_{s} 1_{G_{i}=G_{j}} 1_{i \neq j}+\rho_{m} 1_{G_{i} \neq G_{j}}= \begin{cases}1 & i=j \\ \rho_{s} & i \neq j, G_{i}=G_{j} \\ \rho_{m} & G_{i} \neq G_{j}\end{cases}
$$

where $\rho_{s}, \rho_{m} \in[0,1)$ are some constants provided that $\rho_{s} \geq \rho_{m} \geq \rho_{x z}^{2}$. 
Under the above approximations, the conditional mean and covariance in the prediction (20) are evaluated as follows.

$$
\begin{aligned}
\bar{x}_{t \mid t-1}= & M_{t-1}+K_{t-1} \bar{x}_{t-1 \mid t-1}, \\
\bar{z}_{t \mid t-1}^{i}= & M_{t-1}^{i}+L_{t-1}^{i} \bar{z}_{t-1 \mid t-1}^{i}+K_{t-1}^{i} \bar{x}_{t-1 \mid t-1}, \\
V_{t \mid t-1}= & K_{t-1} K_{t-1} V_{t-1 \mid t-1}+\left(\varsigma_{t-1}\right)^{2} D, \\
V_{t \mid t-1}^{i j}= & L_{t-1}^{i} L_{t-1}^{j} V_{t-1 \mid t-1}^{i j}+K_{t-1}^{i} L_{t-1}^{j} W_{t-1 \mid t-1}^{j}+K_{t-1}^{j} L_{t-1}^{i} W_{t-1 \mid t-1}^{i} \\
& +\varsigma_{t-1}^{i} \varsigma_{t-1}^{j} \Omega^{i j} D, \\
W_{t \mid t-1}^{i}= & K_{t-1} K_{t-1}^{i} V_{t-1 \mid t-1}+K_{t-1} L_{t-1}^{i} W_{t-1 \mid t-1}^{i}+\rho_{x z} \varsigma_{t-1} \varsigma_{t-1}^{i} D .
\end{aligned}
$$

We approximate the yield spread observation equation (14) as follows $^{11}$.

$$
\begin{aligned}
& \tilde{Y}_{t}^{i, n}=Y\left(x_{t}, z_{t}^{i}+\epsilon_{z, t}^{i, n}, \tau_{t}^{i, n}\right)+\tau_{t}^{i, 1}\left(\delta_{l}+\epsilon_{y, t}^{i, n}\right), \\
& \simeq Y\left(\bar{x}_{t \mid t-1}, \bar{z}_{t \mid t-1}^{i}, \tau_{t}^{i, n}\right)+\partial_{x} Y\left(\bar{x}_{t \mid t-1}, \bar{z}_{t \mid t-1}^{i}, \tau_{t}^{i, n}\right)\left(x_{t}-\bar{x}_{t \mid t-1}\right) \\
& +\partial_{z} Y\left(\bar{x}_{t \mid t-1}, \bar{z}_{t \mid t-1}^{i}, \tau_{t}^{i, n}\right)\left(z_{t}^{i}-\bar{z}_{t \mid t-1}^{i}+\epsilon_{z, t}^{i, n}\right) \\
& +\frac{1}{2} \partial_{z z} Y\left(\bar{x}_{t \mid t-1}, \bar{z}_{t \mid t-1}^{i}, \tau_{t}^{i, n}\right)\left(V_{t \mid t-1}^{i i}+\lambda_{z}^{2}\right)+\tau_{t}^{i, n}\left(\delta_{l}+\epsilon_{y, t}^{i, n}\right), \\
& \eta_{t}^{i, n}:=\gamma_{t}^{i, n}\left(\tilde{Y}_{t}^{i, n}-\delta_{l} \tau_{t}^{i, n}-Y\left(\bar{x}_{t \mid t-1}, \bar{z}_{t \mid t-1}^{i}, \tau_{t}^{i, n}\right)\right. \\
& \left.-\frac{1}{2} \partial_{z z} Y\left(\bar{x}_{t \mid t-1}, \bar{z}_{t \mid t-1}^{i}, \tau_{t}^{i, n}\right)\left(V_{t \mid t-1}^{i i}+\lambda_{z}^{2}\right)\right) \\
& \simeq \gamma_{t}^{i, n}\left(\partial_{x} Y\left(\bar{x}_{t \mid t-1}, \bar{z}_{t \mid t-1}^{i, n}, \tau_{t}^{i, n}\right)\left(x_{t}-\bar{x}_{t \mid t-1}\right)\right. \\
& \left.+\partial_{z} Y\left(\bar{x}_{t \mid t-1}, \bar{z}_{t \mid t-1}^{i}, \tau_{t}^{i, n}\right)\left(z_{t}^{i}-\bar{z}_{t \mid t-1}^{i}+\epsilon_{z, t}^{i, n}\right)+\tau_{t}^{i, n} \epsilon_{y, t}^{i, n}\right) \\
& :=H_{t}^{i, n}\left(x_{t}-\bar{x}_{t \mid t-1}\right)+J_{t}^{i, n}\left(z_{t}^{i}-\bar{z}_{t \mid t-1}^{i}\right)+\epsilon_{t}^{i, n} .
\end{aligned}
$$

where,

$$
\gamma_{t}^{i, n}:=\left(\left(\lambda_{z} \partial_{z} Y\left(\bar{x}_{t \mid t-1}, \bar{z}_{t \mid t-1}^{i}, \tau_{t}^{i, n}\right)\right)^{2}+\left(\lambda_{y} \tau_{t}^{i, n}\right)^{2}\right)^{-1 / 2}
$$

and,

$$
\epsilon_{t}^{i, n}:=\gamma_{t}^{i, n}\left(\partial_{z} Y\left(\bar{x}_{t \mid t-1}, \bar{z}_{t \mid t-1}^{i}, \tau_{t}^{i, n}\right) \epsilon_{z, t}^{i, n}+\tau_{t}^{i, n} \epsilon_{y, t}^{i, n}\right),
$$

has unit variance.

With the approximation of $A_{z}\left(z_{t}^{i}, t\right) \simeq A_{z}\left(\bar{z}_{t \mid t-1}^{i}, t\right)$, the conditional mean and covariance in filtering equation (19) are evaluated as follows.

$$
\bar{x}_{t \mid t}=\bar{x}_{t \mid t-1}+V_{t \mid t} \sum_{i \in \mathcal{I}_{t}} \sum_{n \in \mathcal{N}_{t}^{i}} H_{t}^{i, n} \eta_{t}^{i, n}
$$

\footnotetext{
11 We only add the second derivative about $z$, hence the shape of $\hat{Y}(\cdots)$ is strongly curving in the direction $z$. It caused by the definition of $\gamma(\cdots)$.
} 


$$
\begin{aligned}
& +\sum_{i \in \mathcal{I}_{t}} W_{t \mid t}^{i}\left(\lambda_{\xi}\left(\tilde{\xi}_{t}^{i}, t\right)^{-2}\left(\tilde{z}_{t}^{i}-\bar{z}_{t \mid t-1}^{i}\right)+\sum_{n \in \mathcal{N}_{t}^{i}} J_{t}^{i, n} \eta_{t}^{i, n}\right), \\
\bar{z}_{t \mid t}^{i}= & \bar{z}_{t \mid t-1}^{i}+W_{t \mid t}^{i} \sum_{j \in \mathcal{I}_{t}} \sum_{n \in \mathcal{N}_{t}^{j}} H_{t}^{j, n} \eta_{t}^{j, n} \\
& +\sum_{j \in \mathcal{I}_{t}} V_{t \mid t}^{i j}\left(\lambda_{\xi}\left(\tilde{\xi}_{t}^{j}, t\right)^{-2}\left(\tilde{z}_{t}^{j}-\bar{z}_{t \mid t-1}^{j}\right)+\sum_{n \in \mathcal{N}_{t}^{j}} J_{t}^{j, n} \eta_{t}^{j, n}\right), \\
\left(\begin{array}{cc}
V_{t \mid t} & W_{t \mid t}^{j} \\
W_{t \mid t}^{i} & V_{t \mid t}^{i j}
\end{array}\right)^{-1}= & \left(\begin{array}{cc}
V_{t \mid t-1} & W_{t \mid t-1}^{j} \\
W_{t \mid t-1}^{i} & V_{t \mid t-1}^{i j}
\end{array}\right)^{-1}+\lambda_{\xi}\left(\tilde{\xi}_{t}^{i}, t\right)^{-2}\left(\begin{array}{cc}
0 & 0 \\
0_{i} & 1_{i j}
\end{array}\right) \\
& +\left(\begin{array}{cc}
\sum_{k \in \mathcal{I}_{t}} \sum_{n \in \mathcal{N}_{t}^{k}} H_{t}^{k, n} H_{t}^{k, n} & \sum_{n \in \mathcal{N}_{t}^{j}} H_{t}^{j, n} J_{t}^{j, n} \\
\sum_{n \in \mathcal{N}_{t}^{i}} H_{t}^{i, n} J_{t}^{i, n} & 1_{i j} \sum_{n \in \mathcal{N}_{t}^{i}} J_{t}^{i, n} J_{t}^{i, n}
\end{array}\right)
\end{aligned}
$$

where, we regard that $H_{t}^{i, n}=J_{t}^{i, n}=0$ and $\tilde{z}_{t}^{i}-\bar{z}_{t \mid t-1}^{i}=0$ if $i \notin \mathcal{I}_{t}$ or $n \notin \mathcal{N}_{t}^{i}$. And if $\left|\bar{z}_{t \mid t}^{i}\right|>4$, we truncate it.

Since equations (17) and (18), the log likelihood becomes, ${ }^{12}$

$$
\begin{aligned}
\ln P & \left(\tilde{Y}_{t}^{\cdot}, \tilde{\xi}_{t} \mid \tilde{Y}_{\{t-1\}}^{\cdot}, \tilde{\xi}_{\{t-1\}}\right) \\
\simeq & \text { Const }-\frac{1}{2} \ln \left|\left(\begin{array}{cc}
V_{t \mid t-1} & W_{t \mid t-1}^{\cdot} \\
W_{t \mid t-1}^{\cdot} & V_{t \mid t-1}^{\cdot}
\end{array}\right)\right|+\frac{1}{2} \ln \left|\left(\begin{array}{cc}
V_{t \mid t} & W_{t \mid t}^{\cdot} \\
W_{t \mid t}^{\cdot} & V_{t \mid t}^{\cdot !}
\end{array}\right)\right| \\
& +\sum_{i \in \mathcal{I}_{t}} \sum_{n \in \mathcal{N}_{t}^{i}} \ln \left|\gamma_{t}^{i, n}\right|-\sum_{i \in \mathcal{I}_{t}} \ln A_{z}\left(\bar{z}_{t \mid t-1}^{i}, t\right) \\
& +\frac{1}{2}\left(\begin{array}{c}
\bar{x}_{t \mid t}-\bar{x}_{t \mid t-1} \\
\bar{z}_{t \mid t}^{\mid}-\bar{z}_{t \mid t-1}
\end{array}\right)^{T}\left(\begin{array}{cc}
V_{t \mid t} & W_{t \mid t}^{\cdot} \\
W_{t \mid t}^{\cdot} & V_{t \mid t}^{\mid:}
\end{array}\right)^{-1}\left(\begin{array}{c}
\bar{x}_{t \mid t}-\bar{x}_{t \mid t-1} \\
\bar{z}_{t \mid t}-\bar{z}_{t \mid t-1}^{\mid}
\end{array}\right) \\
& -\frac{1}{2} \sum_{i \in \mathcal{I}_{t}} \sum_{n \in \mathcal{N}_{t}^{i}}\left(\eta_{t}^{i, n}\right)^{2}-\sum_{i \in \mathcal{I}_{t}} \frac{1}{2} \lambda_{\xi}\left(\tilde{\xi}_{t}^{i}, t\right)^{-2}\left(\tilde{z}_{t}^{i}-\bar{z}_{t \mid t-1}^{i}\right)^{2} .
\end{aligned}
$$

\subsection{The Result of Data Analysis}

Table I shows our requirement for the data. The sample period is from October 1, 2002 to March 31, 2005 for a total of 613 (time points) $\times$ 183 (entities), 1907 (bonds) observations.

We show the path of the number of bonds in the each duration, spread sector and industrial sector, and at figure 2 . We find that the

\footnotetext{
${ }^{12}$ Hence we assume normality of the observation error, we truncate $\eta_{t}^{\cdot, \cdot}$ and $\tilde{z}_{t}-\bar{z}_{t}$ by upper and lower 5 percentile respectively at each time point $t$ in the estimation of $x_{t \mid t}$ and log likelihood to eliminate the influence of fat tail effect.
} 
distributions of durations and ratings are stable, but the one of spreads is not.

Table I. Conditions of the data

\begin{tabular}{c|c}
\hline Rating & better than R\&I BBB- \\
Duration & from 1 year to 11 years \\
Spread & less than 5\% \\
\hline
\end{tabular}

$$
\begin{aligned}
& \text { Put }\left\{\zeta_{\mathrm{BBB}-}, \zeta_{\mathrm{AAA}}\right\}=\{0,1, \ldots, 9\}^{13}, \text { and } \\
& \bar{z}(\xi, t):= \#\left\{\xi_{t}^{i}=\xi ; i \in \mathcal{I}_{t}\right\}^{-1}\left(\sum_{i \in \mathcal{I}_{t}, \xi_{t}^{i}=\xi} \bar{z}_{t \mid t}^{i}\right), \\
& V_{z}(\xi, t+1):=\left(\begin{array}{c}
0 \\
1_{\xi_{t}^{i}=\xi}
\end{array}\right)\left(\begin{array}{cc}
V_{t \mid t} & W_{t \mid t}^{\cdot} \\
W_{t \mid t} & V_{t \mid t}^{-1}
\end{array}\right)^{-1}\left(\begin{array}{c}
0 \\
1_{\xi_{t}^{j}=\xi}
\end{array}\right) \\
&+\left(\#\left\{\xi_{t}^{i}=\xi: i \in \mathcal{I}_{t}\right\}-1\right)^{-1} \sum_{i \in \mathcal{I}_{t}, \xi_{t}^{i}=\xi}\left(\bar{z}_{t \mid t}^{i}-\bar{z}(\xi, t)\right)^{2} .
\end{aligned}
$$

But if $V_{z}(\xi, t) \leq 0.01^{2}$, we put, $V_{z}(\xi, t)=0.01^{2}$. We estimate the mapping function $\psi(\xi, t)$ as the least-squares spline approximation of $\bar{z}_{i}(\xi, t-1)$ with the weight $1 / V_{z}(\xi, t)^{2}$, in the range of $[-4,4]$. Figure 3 shows the estimated result of $\psi(\xi, t)$. Meanwhile, we put,

$$
\lambda_{z}(\xi, t):=\frac{1}{2 \partial_{\xi} \psi(\xi, t)} .
$$

In our model the mapping function is not static. It represents typical credit reliability of each credit rating at each date. In whole of observation period, the mapping functions are almost in the upward trend. It is significantly at the middle range. The highest CQI is limited about 3 . So the range of the mapping becomes narrow. It just corresponds to the tightening of the credit spreads. From July to September 2003, we find a short downward trend.

Table II shows the maximum likelihood estimation of the model parameters. We ignore the likelihood at the first 20 business days to eliminate the influence of the initial value. The prior distribution of

\footnotetext{
13 To justify the approximation about $A_{z}(z, t)$, we should implicitly consider outer credit ratings which are never observed.
} 
state variables are approximated by multi dimensional normal distribution which has same mean and covariance matrix with unconditional distribution of them.

We list the summary of the estimation below.

$-\mathrm{MCI}$

- The reverting mean of MCI are larger in the pricing measure $Q$, compared with the one in the physical measure $P$. $\left(\tilde{\theta_{x}}=\right.$ $\left.64.218, \theta_{x}=30.094\right)$.

- The time constant of mean reversion of MCI is about $1 / \kappa_{x}=$ 3.4977 [year].

- The typical uncertainty of MCI is about $\sqrt{\sigma_{x}^{2} \theta_{x} / 2 \kappa_{x}}=17.025$.

$-\mathrm{CQI}$

- The reverting mean of CQI are larger in the physical measure $P$, compared with the one in the pricing measure $Q\left(\theta_{z}=0\right.$, $\left.\tilde{\theta}_{z}=-1.2714\right)$.

- The time constant of mean reversion of CQI is about $1 / \kappa_{z}=$ 44.490 [year].

- The typical uncertainty of CQI is 1.

- Correlation

- There exists positive correlation between the fluctuations of CQIs each other. $\left(\rho_{s}\right.$ and $\left.\rho_{m}\right)$

- There exists negative, but very small correlation between the fluctuation of CQIs and MCI $\left(\rho_{x z}\right)$.

- The industrial sector affect to the correlations between the fluctuations of CQIs.

- The correlation parameter $\rho_{s}$ and $\rho_{m}$ between the fluctuations of CQIs each other are large compared to the required value from the correlation parameter of CQIs and MCI, $\left|\rho_{x z}\right|^{2}=$ 0.00121 .

- Hazard function

- $\quad$ See figure 4 .

- Observation

- $\lambda_{z}$ is significantly small compared with the typical uncertainty of CQI and the value range of mapping function. 
- The liquidity spread is about $\delta_{l}=6.8266$ [bps].

Both of the reverting mean of MCI and CQI are acceptable and they are more risky (i.e. cause the higher hazard rate) on measure $Q$ than on measure $P$.

The time constants give the typical time scales about memory of present values. The results shows that the influence of present condition remains about 3.5 years in the dynamics of the whole market and those remains about 44 years in the credit quality dynamics of individual entities. These results are realistic in practice.

Hence $\lambda_{z}$ and $\lambda_{y}$ are small, we find that the CQIs are tightly related to the yield spreads.

Figure 4 shows the estimation of loss-adjusted hazard rate, $\gamma(x, z)$. It shows the well known fact that the gradients of spreads are steep at low credit ratings.

Figure 4 shows the estimation of YSTQS at each MCI equals to 20, 40,60 , or 80 . These graphs match to the $\gamma(x, z)$ at the point of zero duration hence the terminal condition of $Y(x, z, \tau)$. At the long duration region, those graphs are almost same, hence the mean reversion property of MCI. Besides, the details of short duration region vanish at the long duration region; it is caused by the diffusion of CQI.

Figure 6 shows the yield spread term structure of obligor who's CQIs is 3.0, 1.5, 0.5, or -0.5 respectively, when the MCI is 20, 40, 60, or 80 . We note the typical credit rating corresponds to each CQI as a reference. Strong normal yields are observed in the graphs of high MCI. The mean reversion of MCI, which has small time constant (3.5 years) generates the gradient of term structures. Besides the mean reversion property of CQI generates normal yields at higher CQI. But we find a reversal that it is stronger in the graph $\mathrm{CQI}=0.5$ than $\mathrm{CQI}=1.5$. We guess that this is caused by the shape of hazard rate which is rather flat at $\mathrm{CQI}=1.0$.

Let us consider the path of state variables. Figure 7 shows the estimated path of MCI. We regard that it represents gradient of yield spread term structure; i.e., the high (resp. low) MCI corresponds to the flat (resp. steep) term structure. We see that MCI was in the downward trend (steepening) at first, but after August 2004, it was in upward trend (flattening).

Hence it is difficult to show the estimations of the whole entities in this short article, we choose typical 8 entities from their average of CQI in the period. Figure 8 shows the comparison between the filtered estimation of CQIs $\bar{z}_{t \mid t}^{i}$, and the credit ratings translated to the CQI space $\tilde{z}_{t}^{i}$. We can see that the credit ratings and CQIs are almost same 
level in rough, but they are not same in details. This means that the credit ratings can explain rough level of yield spreads with dynamical mapping function.

Figure 9 shows the path of yield spread of the bond which has maximum and minimum spread issued by each entity. Two dushed lines in each graph show the estimation by our model and the two solid lines show the observation respectively. The results are seems to be good except code 7201 . There observed a reverse yield in early period, and it is hard to explain. We consider it might be a miss pricing in the market. The rest shows that our model represents the term structures of many entities with one common factor (MCI) and one individual factor (CQI) satisfactory. Figure 10 shows the path of theoretical yield spread of each entity at several term to maturity.

\section{Conclusion}

We modeled the loss-adjusted hazard rates as a function of the Macro Credit Indicators (MCIs) which describes the fluctuation of the whole corporate bond market, and Credit Quality Indicators (CQIs) which describe the credit reliability of individual issuer. We showed that the prices of defaultable bonds are represented by the Yield Spread TermQuality Surface (YSTQS) in our model and derived the shape and dynamics of YSTQS.

We examined our model with daily spread and credit rating data, and estimated the histrical path of MCI and CQIs. The estimated CQI tells us the credit reliability implied in the yield spread for the individual issuer. On the other hand, MCI tells us the economic condition. And we showed that the market requires some premiums for the risk of MCI and CQIs.

We can draw the theoretical yield spread term structure of each entities which match the observed yield spreads and natural to each other in spite of there are not sufficient issue of bonds with individual entities. Our model tells us the joint dynamics of the yield spread term structure of whole market. This means that we can control the spread risk of plural entities consistently. 
Table II. Estimation of parameters

\begin{tabular}{|c|c|c|}
\hline MCI $x_{t}$ & $\begin{array}{c}\kappa_{x} \\
\theta_{x} \\
\tilde{\theta}_{x} \\
\sigma_{x}\end{array}$ & $\begin{array}{r}0.28590 \\
64.218 \\
30.094 \\
2.3467\end{array}$ \\
\hline $\mathrm{CQI} z_{t}^{i}$ & $\begin{array}{l}\kappa_{z} \\
\theta_{z} \\
\tilde{\theta}_{z}\end{array}$ & $\begin{array}{r}0.022477 \\
0^{*} \\
-1.2714\end{array}$ \\
\hline correlation & $\begin{array}{l}\rho_{s} \\
\rho_{m} \\
\rho_{x z}\end{array}$ & $\begin{array}{r}0.46839 \\
0.35869 \\
-0.034754\end{array}$ \\
\hline hazard function & $\begin{array}{c}x_{0} \\
z_{0} \\
\gamma_{0} \\
\phi(4.00) \\
\phi(2.00) \\
\phi(0.00) \\
\phi(-2.00) \\
\phi(-4.00)\end{array}$ & $\begin{array}{r}100^{*} \\
0.0^{*} \\
0.01^{*} \\
-8.3819 \\
-2.4673 \\
-1.5506 \\
1.5132 \\
1.5148\end{array}$ \\
\hline observation & $\begin{array}{l}\lambda_{y} \\
\lambda_{z} \\
\delta_{l} \\
\Delta\end{array}$ & $\begin{array}{r}0.94756[\mathrm{bps}] \\
0.028445 \\
6.8266[\mathrm{bps}] \\
1 / 250 \text { [year] }^{*}\end{array}$ \\
\hline
\end{tabular}
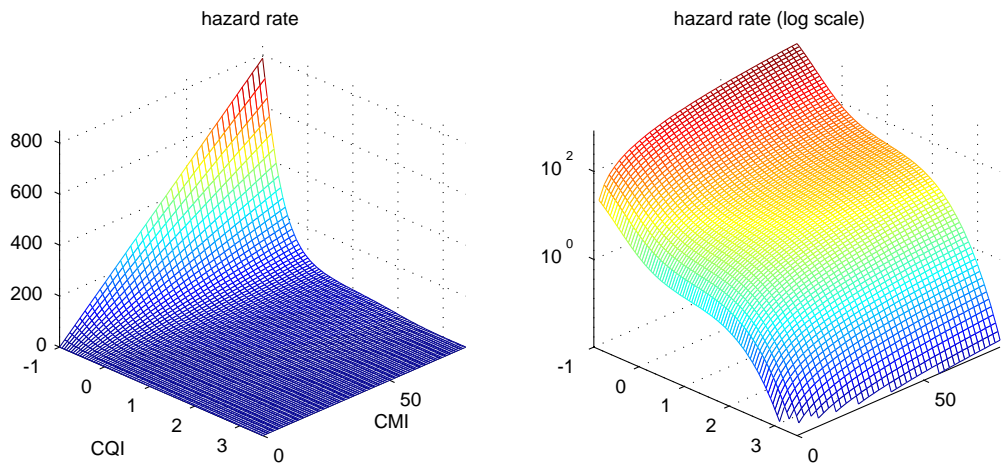

Figure 4. Estimated loss-adjusted hazard rate function, $\gamma(x, z)$ [bps] 

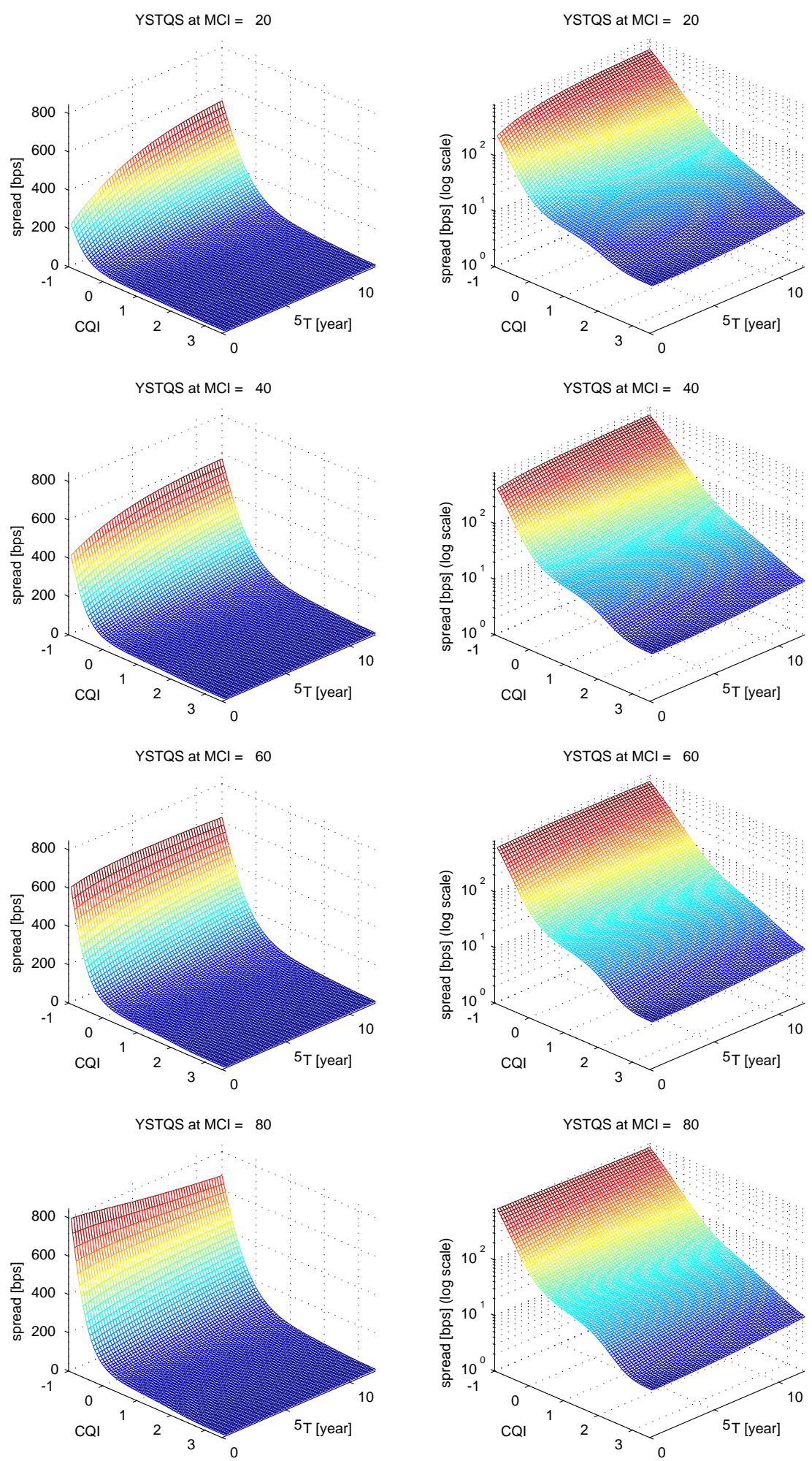

Figure 5. Estimated Yield Spread Term-Quality Surface [bps] (left:linear-scale, right:log-scale) 

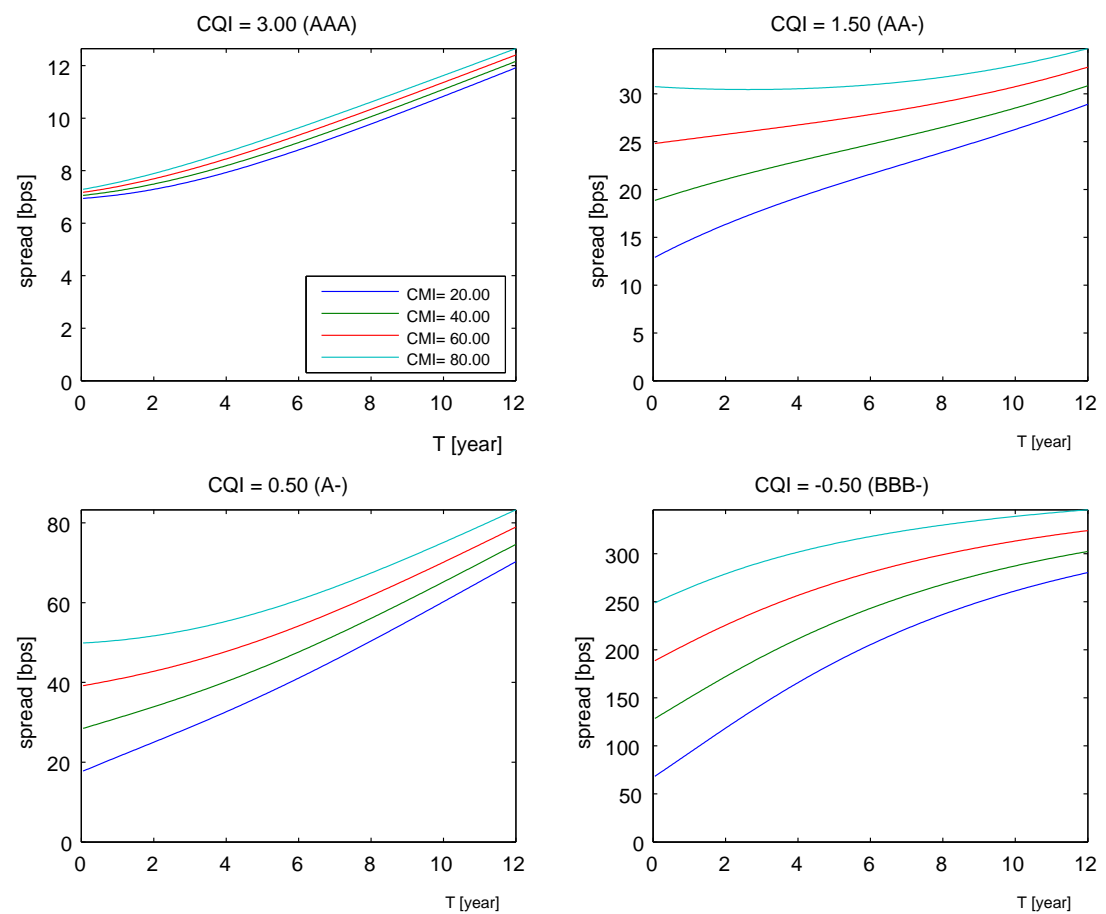

MCI $=80,60,40,20$, in order starting with the upper line at each figure.

Figure 6. MCI dependence of Yield Spread Term Structure at each CQI [bps]

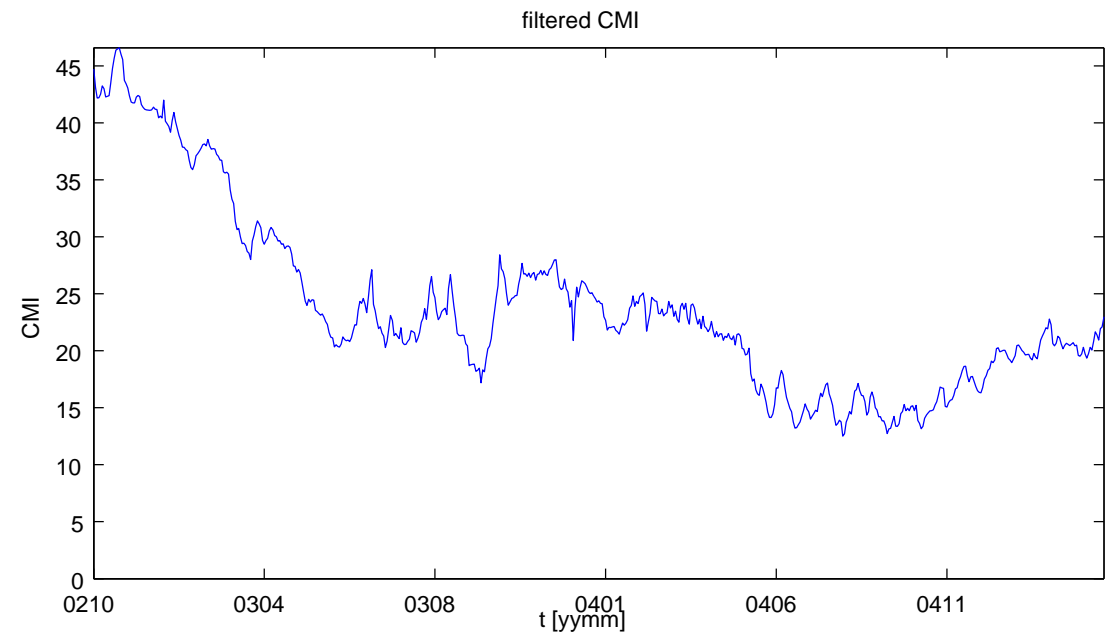

Figure \%. Filtered mean of MCI 

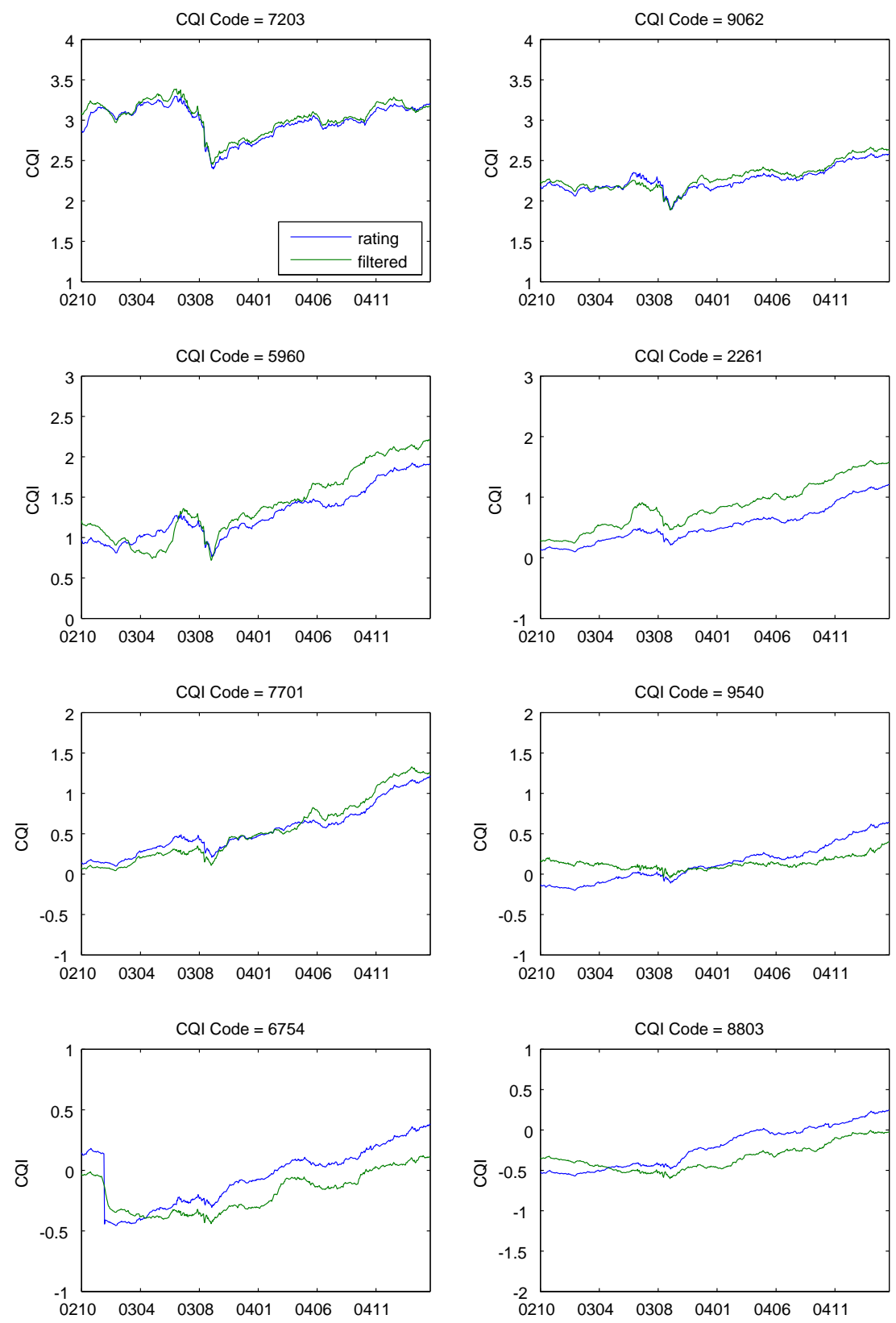

Figure 8. Observed and Filtered CQIs 

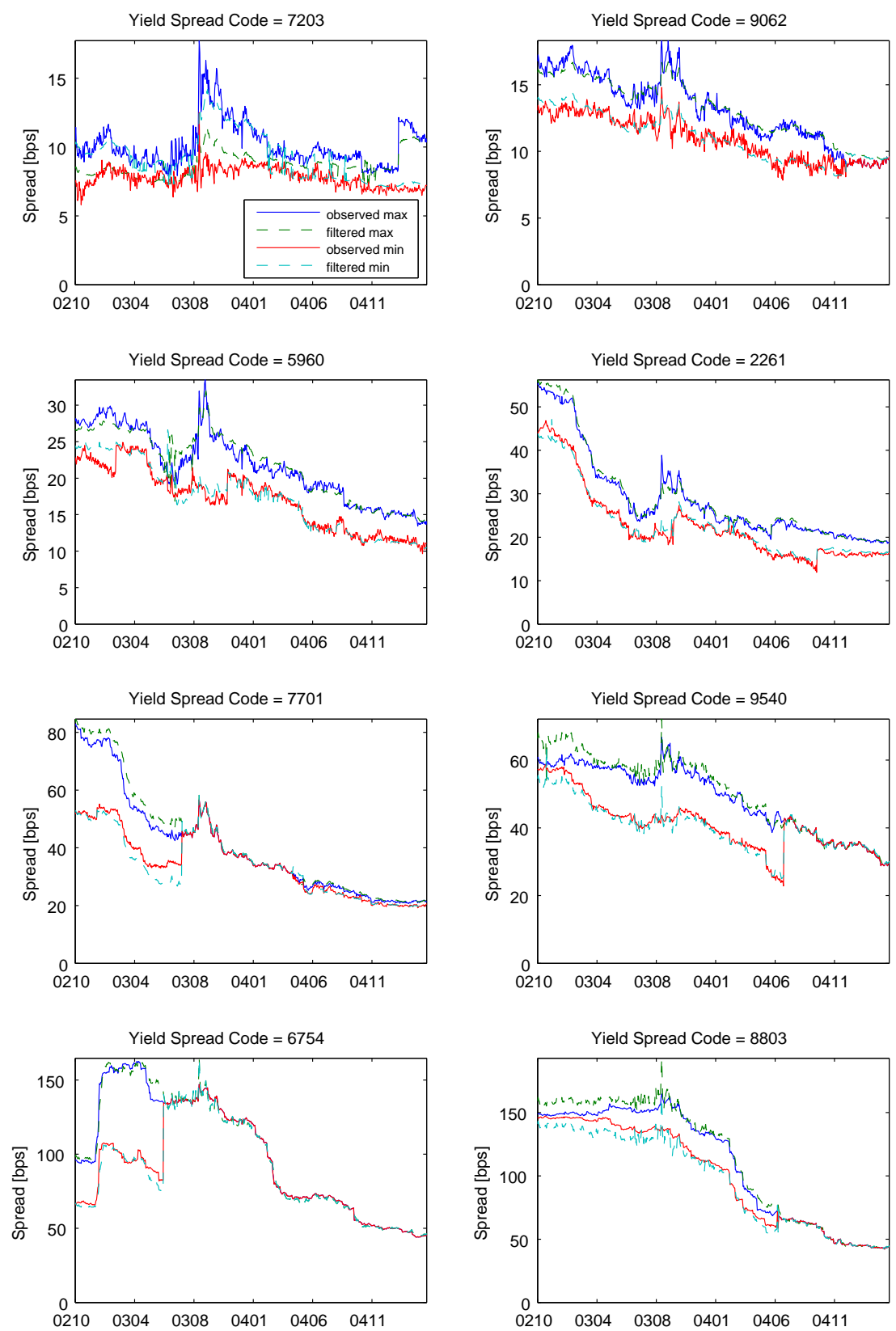

Figure 9. Observed and Filtered Yield Spread (maximam and minimum for each entity) 

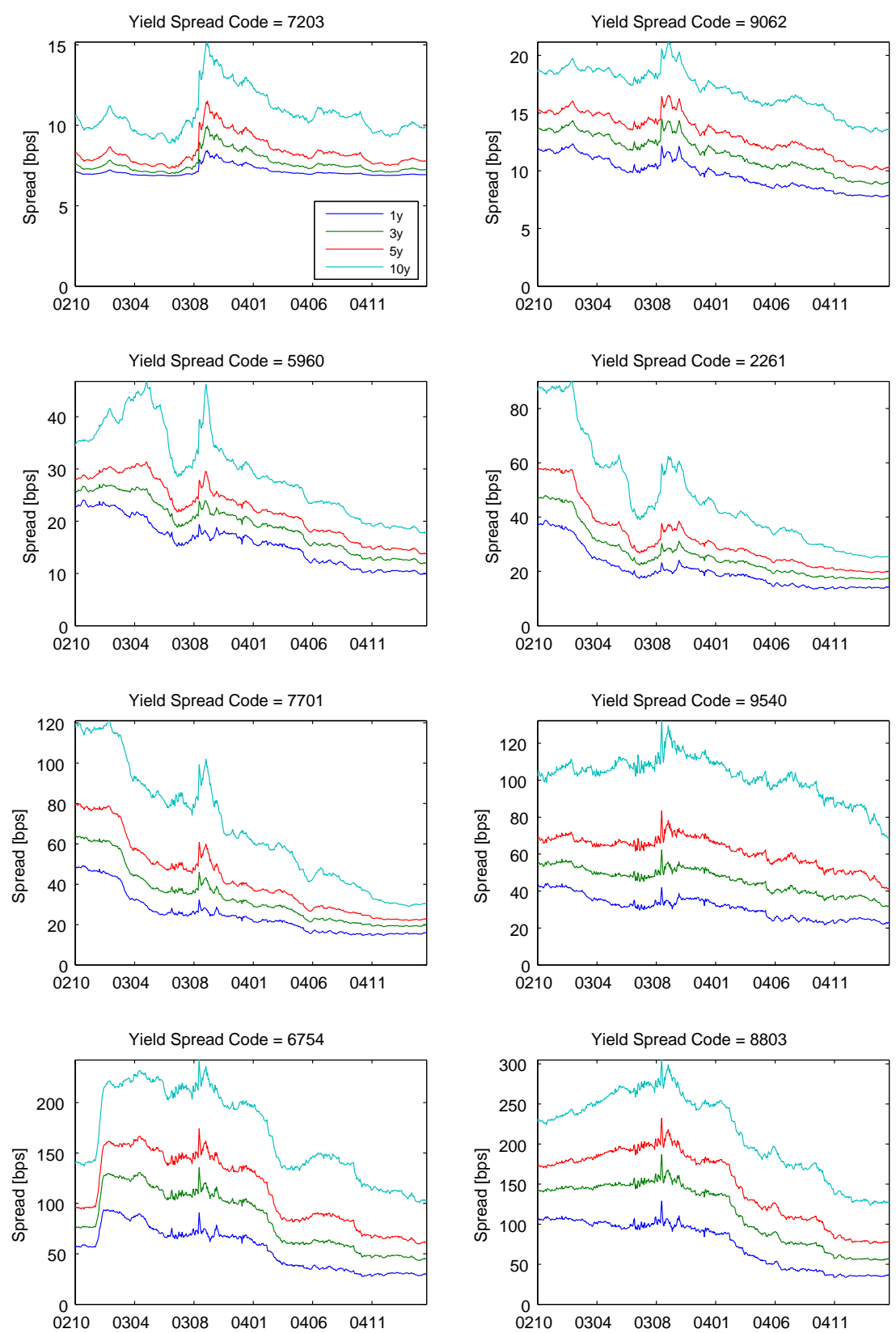

Figure 10. Estimated Yield Spreads ( 1, 3, 5, 10 [year] ) 


\section{Appendix}

\section{A. Proof of proposition 2}

Likewise the case of default indicator, hence the $N_{t}^{i} L_{i}$ is a submartingale, there exists $\left(\mathcal{F}_{t}^{M}\right)$-predictable right continuous nonnegative monotonically nondecreasing process $A_{t}^{i}$ where $A_{0}^{i}=0$ and $N_{t}^{i} L_{i}-A_{t}^{i}$ is a $\left(Q,\left(\mathcal{F}_{t}^{M}\right)\right)$-martingale.

LEMMA 14. If the assumption 1 hold, cumulative loss-adjusted hazard process $A_{t}^{i}$ is absolutely continuous in $t \in[0, T]$.

Proof. $N_{t}^{i}\left(1-L_{i}\right)$ is a submartingale. From the uniqueness of the Doob-Meyer's decomposition, we find that $\tilde{\Lambda}_{t}^{i}-A_{t}^{i}$ is a $\left(\mathcal{F}_{t}^{M}\right)$-predictable, right continuous nonnegative monotonically nondecreasing process, i.e., for any $u \leq t \leq T$,

$$
\tilde{\Lambda}_{t}^{i}-A_{t}^{i} \geq \tilde{\Lambda}_{u}^{i}-A_{u}^{i} .
$$

Recall that both of $\tilde{\Lambda}_{t}^{i}$ and $A_{t}^{i}$ are nondecreasing, so the following inequality should hold.

$$
\left|\tilde{\Lambda}_{t}^{i}-\tilde{\Lambda}_{u}^{i}\right| \geq\left|A_{t}^{i}-A_{u}^{i}\right| .
$$

Because, $\tilde{\Lambda}_{t}^{i}$ is absolutely continuous, so is $A_{t}^{i}$.

Q.E.D.

Therefore $A_{t}^{i}$ is represented by some $\left(Q,\left(\mathcal{F}_{t}^{M}\right)\right)$-adapted process $\gamma_{t}^{i}$ as follows.

$$
A_{t}^{i}=\int_{0}^{t} d s\left(1-N_{s-}^{i}\right) \gamma_{s}^{i}
$$

where $\left(1-N_{t-}^{i}\right) \gamma_{t}^{i} \geq 0$. Moreover, hence $\tilde{\Lambda}_{t}^{i}-A_{t}^{i}$ is a monotonically nondecreasing process, we find that $\left(1-N_{t-}^{i}\right)\left(\tilde{h}_{t}^{i}-\gamma_{t}^{i}\right) \geq 0$.

\section{B. Proof of Theorem 3}

Define $M_{t, T}^{i}$ as a $\left(Q,\left(\mathcal{F}_{t}^{M}\right)\right)$-martingale on $t \in[0, T]$ from terminal prices of defaultable bonds as follows.

$$
M_{t, T}^{i}:=E^{Q}\left[\left(1-N_{T}^{i}\right) S_{T}^{-1}+R_{T, T}^{i} S_{T \wedge \tau_{i}}^{-1} \mid \mathcal{F}_{t}^{M}\right] .
$$

And the price of corporate bonds are represented as follows,

$$
p_{t, T}^{i}=\left(1-N_{t-}^{i}\right) M_{t, T}^{i} S_{t} .
$$


LEMMA 15. For any $i=1, \ldots, N, T \in[0, \bar{T}], t \in[0, T]$, the following equations should hold.

$$
\begin{aligned}
R_{t, T}^{i} & =N_{t}^{i} M_{t \wedge \tau_{i}, T}^{i} S_{t \wedge \tau_{i}}, \\
\Delta N_{t}^{i} \Delta M_{t, T}^{i} & =\Delta N_{t}^{i}\left(R_{t, T}^{i} S_{t}^{-1}-M_{t-, T}^{i}\right)=\Delta N_{t}^{i} M_{t-, T}^{i} L_{i}, \\
p_{t, T}^{i} & =\left(1-N_{t}^{i}\right) M_{t, T}^{i} S_{t}+\left(1-N_{t-}^{i}\right) R_{t, T}^{i} .
\end{aligned}
$$

Proof. Equations (28) and (29) are easily derived from the definition of $M_{t, T}^{i}$ and $R_{t, T}^{i}$ and that $N_{t} N_{u}=N_{t \wedge u}, \Delta N_{t}^{i}=1_{t=\tau_{i}} \Delta N_{t}^{i}$.

(30) is derived directly from (28) and,

$$
p_{t, T}^{i}=\left(1-N_{t-}^{i}\right) M_{t, T}^{i} S_{t}=\left(1-N_{t}^{i}\right) M_{t, T}^{i} S_{t}+\Delta N_{t}^{i} M_{t, T}^{i} S_{t} .
$$

Q.E.D.

LEMMA 16. For any $i=1, \ldots, N, T \in[0, \bar{T}], t \in[0, T]$, define,

$$
\tilde{M}_{t, T}^{i}:=e^{-A_{t}^{i}} M_{t \wedge \tau_{i}-, T}^{i} .
$$

Then, $\tilde{M}_{t, T}^{i}$ is a $\left(Q,\left(\mathcal{F}_{t}^{M}\right)\right)$-martingale.

Proof. Applying Ito formula, we have,

$$
\begin{aligned}
\tilde{M}_{t, T}^{i}= & \left(1-N_{t}^{i}\right) e^{-A_{t}^{i}} M_{t, T}^{i}+N_{t}^{i} e^{-A_{\tau_{i}}^{i}} M_{\tau_{i}-, T}^{i} \\
= & -\int_{0}^{t} d A_{u}^{i} e^{-A_{u}^{i}} M_{u-, T}^{i}+\int_{0}^{t}\left(1-N_{u-}^{i}\right) e^{-A_{u}^{i}} d M_{u, T}^{i} \\
& -\sum_{u \leq t} e^{-A_{u}^{i}} \Delta N_{u}^{i} \Delta M_{u, T}^{i}-\sum_{u \leq t} \Delta N_{u}^{i}\left(e^{-A_{u}^{i}} M_{u-, T}^{i}-e^{-A_{\tau_{i}}^{i}} M_{\tau_{i}-, T}^{i}\right) \\
= & \int_{0}^{t} e^{-A_{u}^{i}}\left(M_{u-, T}^{i}\left(L_{i} d N_{u}^{i}-d A_{u}^{i}\right)+\left(1-N_{u-}^{i}\right) d M_{u, T}^{i}\right) .
\end{aligned}
$$

where we use equation (29). The right hand side of the above equation is a $\left(Q, \mathcal{F}_{t}^{M}\right)$-local martingale. Since $\tilde{M}_{t, T}^{i}$ is bounded, it is a $\left(Q, \mathcal{F}_{t}^{M}\right)$ martingale.

Q.E.D.

Now we try to prove theorem 3. From equation (30) and (31), the condition (2) is equivalent to,

$$
\left(1-N_{t}^{i}\right) X_{t, T}^{i}=\left(1-N_{t}^{i}\right) \tilde{M}_{t, T}^{i} .
$$


First, we assume that equation (32) holds at $t \in[0, T]$. Obviously, condition of equation (4) holds. And $\hat{M}_{t, T}^{i}=X_{t, T}^{i}=\tilde{M}_{t, T}^{i}$ at $t<\tau_{i}$.

From the definition, $N_{t}^{i} \tilde{M}_{t, T}^{i}=N_{t}^{i} \tilde{M}_{\tau_{i}-, T}^{i}$ and $N_{t}^{i} \hat{M}_{t, T}^{i}=N_{t}^{i} \hat{M}_{\tau_{i}-, T}^{i}$, i.e., the both of $\tilde{M}_{t, T}^{i}$ and $\hat{M}_{t, T}^{i}$ freeze after the default event. So we find that $\hat{M}_{t, T}^{i}=\hat{M}_{\tau_{i}-, T}^{i}=\tilde{M}_{\tau_{i}-, T}^{i}=\tilde{M}_{t, T}^{i}$ at $t \geq \tau_{i}$. So $\hat{M}_{t, T}^{i}$ is a $\left(Q,\left(\mathcal{F}_{t}^{M}\right)\right)$-martingale.

Second, we assume that $\hat{M}_{t, T}^{i}$ is a $\left(Q, \mathcal{F}_{t}^{M}\right)$-martingale, and equations (4) holds. Define,

$$
\eta_{t, T}^{i}:=\left(1-N_{t}^{i}\right) e^{\tilde{\Lambda}_{t}^{i}}\left(\tilde{M}_{t, T}^{i}-\hat{M}_{t, T}^{i}\right), \quad t \in[0, T] .
$$

Hence,

$\eta_{t, T}^{i}=\int_{0}^{t}\left(1-N_{s-}^{i}\right) e^{\tilde{\Lambda}_{s}^{i}}\left(\left(d \tilde{M}_{s, T}^{i}-d \hat{M}_{s, T}^{i}\right)-\left(\tilde{M}_{s-, T}^{i}-\hat{M}_{s-, T}^{i}\right)\left(d N_{s}^{i}-d \tilde{\Lambda}_{s}^{i}\right)\right)$,

we see that $\eta_{t, T}^{i}$ is a $\left(Q,\left(\mathcal{F}_{t}^{M}\right)\right)$-local martingale. Since $E^{Q}\left[\sup _{s \leq T}\left|\eta_{s, T}^{i}\right|\right]<$ $\infty$, it is a $\left(Q,\left(\mathcal{F}_{t}^{M}\right)\right)$-martingale ${ }^{14}$. Therefore,

$$
\left(1-N_{t}^{i}\right)\left(\tilde{M}_{t, T}^{i}-\hat{M}_{t, T}^{i}\right)=e^{-\tilde{\Lambda}_{t}^{i}} \eta_{t, T}^{i}=e^{-\tilde{\Lambda}_{t}^{i}} E^{Q}\left[\eta_{T, T}^{i} \mid \mathcal{F}_{t}^{M}\right]=0 .
$$

This means that equation (32) holds.

Note that we defined three kinds of stochastic processes, $M_{t, T}^{i}, \tilde{M}_{t, T}^{i}$ and $X_{t, T}^{i}$ to express the defaultable bond prices. The typical properties of them are summarized in table III.

Table III. property of $M_{t, T}^{i}, \tilde{M}_{t, T}^{i}$ and $X_{t, T}^{i}$

\begin{tabular}{c|cc} 
& at default time & after the default time \\
\hline$M_{t, T}^{i}$ & jump & freeze \\
$\tilde{M}_{t, T}^{i}$ & no jump & freeze \\
$X_{t, T}^{i}$ & no jump & free \\
\hline
\end{tabular}

\section{References}

Black, F., and J. C. Cox (1976) "Valuing corporate securities: Some effects of bond indenture provisions," Journal of Finance, 31, 351-367.

\footnotetext{
14 See Protter (2003) p.38 Theorem 51.
} 
Blanchet-Scallied, C. and M. Jeanblanc (2004) "Hazard rate for credit risk and hedging defaultable contingent claims," Finance and Stochastics, 8, 145-159.

Bielecki, T., S. Crepéy, M. Jeanblanc, and M. Rutkowski (2005) "Valuation of Basket Credit Derivatives in the Credit Migrations Environment," Working Paper.

Bielecki, T., and M. Rutkowski (2001) "Credit Risk: Modeling, Valuation and Hedging," Springer Finance.

Constantindes, G. M. (1992) "A Theory of the Nominal Term Structure of Interest Rates," Review of Financial Studies, 5, 4, 531-552.

Douady, R. and M. Jeanblanc (2002) "A Rating-Based Model for credit Derivatives," European Investment Review www,theeir.com.

Duffie, D., and R. Kan (1996) "A Yield-Factor Model of Interest Rates," Mathematical Finance, 6, 379-406.

Duffie, D., D. Lando (2001) "Term Structure of Credit Spreads with Incomplete Accounting Information," Econometrica, 69, 633-664.

Duffie, D., and K. Singleton (1999) "Modeling Term Structures of Defaultable Bonds," Review of Financial Studies, 12, 687-720.

Duffee, G. R. (1999) "Estimating the Price of Default Risk," Review of Financial Studies, Vol.12, 1, 197-226.

Farnsworth, H., and T. Li (2003) "Modeling Credit Spreads and Ratings Migration," Working Paper.

Feldhütter, P., and D. Lando (2005) "Decomposing Swap Spreads," Working Paper.

Heath, D., R. Jarrow, and A. Morton (1992) "Bond Pricing and the Term Structure of Interest Rates: A New Methodology for Contingent Claims Valuation," Econometrica, 60, (1), 77-105.

Jarrow, R., D. Lando, and S. Turnbull (1997) "A Markov Model for the Term Structure of Credit Risk Spreads," Review of Financial Studies, 10, 481-523.

Jamshidian, F. (1996) "Bond, futures and option valuation in the quadratic interest rate model," Applied Mathematical Finance, 3, 93-115.

Kusuoka, S. A. (1999) "remark on default risk models," Advances in Mathematical Economics, 1, 69-82.

Merton, R. (1974) "On the Pricing of Corporate Debt: The Risk Structure of Interest Rates," Journal of Finance, 29, 449-470.

Protter, P. (2003) Stochastic Integration and Differential Equations: 2nd Edition, Springer-Verlag, New York.

Shouda, T. (2005) "Dynamical Analysis of the Yield Spread Term-Quality Surface Defined on the Duration - Credit Quality Space," Asia-Pacific Financial Markets, (forthcoming). 
